NBER WORKING PAPER SERIES

\title{
HEDGING SUDDEN STOPS AND PRECAUTIONARY RECESSIONS: A QUANTITATIVE FRAMEWORK
}

\author{
Ricardo J. Caballero \\ Stavros Panageas \\ Working Paper 9778 \\ http://www.nber.org/papers/w9778 \\ NATIONAL BUREAU OF ECONOMIC RESEARCH \\ 1050 Massachusetts Avenue \\ Cambridge, MA 02138 \\ June 2003
}

We are grateful to Victor Chernozhukov, Gordon Hanson, Arvind Krishnamurthy, an anonymous referee, and seminar participants at MIT, UTDT, and the AEA meetings for their comments, to Andrei Levchenko for excellent research assistance, and to Alejandro Izquierdo for providing us the data on sudden stops from Calvo et al (2004). Caballero thanks the NSF for financial support. The views expressed herein are those of the authors and not necessarily those of the National Bureau of Economic Research.

(O2003 by Ricardo J. Caballero and Stavros Panageas. All rights reserved. Short sections of text not to exceed two paragraphs, may be quoted without explicit permission provided that full credit including $\odot$ notice, is given to the source. 
Hedging Sudden Stops and Precautionary Recessions: A Quantitative Framework

Ricardo J. Caballero and Stavros Panageas

NBER Working Paper No. 9778

June 2003, Revised July 2006

JEL No. E2, E3, F3, F4, G0, C1

\begin{abstract}
Even well managed emerging market economies are exposed to significant external risk, the bulk of which is financial. At a moment's notice, these economies may be required to reverse the capital inflows that have supported the preceding boom. While capital flows crises are sudden nonlinear events (sudden stops), their likelihood fluctuates over time. The question we address in the paper is how should a country react to these fluctuations. Depending on the hedging possibilities the country faces, the options range from pure self-insurance to hedging the sudden stop jump itself. In between, there is the more likely possibility to hedge the smoother fluctuations in the likelihood of sudden stops. The main contribution of the paper is to provide an analytically and empirically tractable model that allows us to characterize and quantify optimal contingent liability management in a variety of scenarios. We show, with a concrete example, that the gains from contingent liability management can easily exceed the equivalent of cutting a country's external liabilities by 10 percent of GDP.
\end{abstract}

Ricardo J. Caballero

Department of Economics

MIT, 50 Memorial Drive

E52-252 2326 SH-DH

Cambridge, MA 02142

and NBER

caball@mit.edu

Stavros Panageas

The Wharton School

University of Pennsylvania

3620 Locust Walk

Philadelphia, PA 19106

panageas@wharton.upenn.edu 


\section{Introduction}

Even well managed emerging market economies are subject to an occasional "sudden stop" of net capital inflows. That is, at a moment's notice these economies may be required to reverse the capital inflows that supported the preceding boom.

The deep contractions triggered by this sudden tightening of external financial constraints have great costs for these economies, and therefore represent one of the main macroeconomic policyproblems for emerging market economies. There are many domestic and external factors behind these sudden stops in net capital inflows. While there is substantial agreement on the kind of policy adjustments that reduce domestic risks, there is less consensus on what are the adequate external liability management strategies to deal with external shocks. In Caballero and Panageas (2005) we analyzed reserves accumulation and hedging mechanisms to soften the impact of sudden stops once these take place. In this paper we focus on a different aspect of the problem: We argue that there are substantial fluctuations in the likelihood of a sudden stop, and that in many instances these fluctuations are correlated with persistent external variables which offer hedging possibilities free of moral hazard or (emerging market specific) informational concerns (see Sections 5 and 6 for evidence).

The main technical contribution of the paper is a model that is stylized enough to allow extensive analytical characterization, but is also flexible and realistic enough to generate quantitative guidance. The model has two central features: First is the sudden stop, which we characterize as a probabilistic event that, once triggered, requires the country to reduce the pace of external (net) borrowing significantly. This specification of sudden stops captures well policymakers' concerns not only with the stock of external liabilities but also with the size of current account deficits (a flow variable). Second is a signal, which describes the likelihood of sudden stop at each point in time. We characterize the impact of changes in this signal on optimal precautionary savings, and the role of different hedging opportunities in reducing the extent of fluctuations due to precautionary actions and the direct cost of sudden stops.

On one extreme, if the country has no access to hedging instruments, the only tool at hand is a sort of precautionary contraction. As external indicators of the likelihood of a sudden stop worsen, the country slows down the pace of borrowing. Of course, in reality many countries choose 
to ignore these signals and experience deeper contractions once sudden stops take place. Others are excessively prudent, and tighten monetary and fiscal policy beyond what many see as reasonable. The first of our goals in this paper is normative, and it is to take a step in the direction of offering an empirically based metric to evaluate how much prudence is reasonable.

On the other extreme is the case where the country can hedge both the (jump) sudden stop directly and (diffusive) fluctuations in the price of such hedge as the signal fluctuates. In this case the country has maximal insurance. However, since our constraint is directly on the current account deficits, such insurance does not remove sudden stop entirely but only reduces the country's level of external liabilities at the onset of the sudden stop, at minimal cost. What it does do, is to remove the need for precautionary contractions and it limits the extent of a sudden stop to a level that removes jumps in the value of a marginal dollar at the time of the sudden stop (since the reduction in external liabilities raises utility in the post-sudden stop stage enough to compensate for the welfare loss from reduced consumption during the sudden stop).

In an intermediate range, is the more feasible scenario where the jump cannot be hedged but smooth diffusive hedging is available. In this case the country still can remove the bulk of precautionary contractions and reduce the cost of sudden stops, but it remains exposed to the realization of the latter. One of the quantitative contributions of the paper is to show that this type of hedging can go a long way in reducing the overall costs of living in a sudden stop prone environment.

The case of Chile, a prudent emerging market economy, provides a useful framework of reference. Chile's business cycle is highly correlated with the price of copper, its main export good; so much so that this price has become a signal of aggregate Chilean conditions to (foreign and domestic) investors and policymakers alike. As a result of the many internal and external reactions to this signal, the decline in Chilean economic activity when the price of copper falls sharply is many times larger than the annuity value of the wealth effect of the price decline. This contrasts with the scenario in Australia, a developed economy not exposed to the possibility of a sudden stop, where similar terms-of-trade shocks are fully absorbed by the current account, with almost no impact on domestic activity and consumption. ${ }^{1}$ Chile is a natural candidate for the type of hedging strategies we propose.

We do not attempt to explain why international financial markets treat Chile and Australia

\footnotetext{
${ }^{1}$ See , e.g., Caballero (2001).
} 
so differently, or even why the signal for the sudden stops should be so correlated with the price of copper. Instead, we take the "sudden stops" feature as a description of the environment and characterize the optimal risk management strategy under different assumptions about imperfections in hedging markets. ${ }^{2}$

We estimate Chile's probability of facing a sudden stop as a function of the price of copper and calibrate the parameters to match observed consumption fluctuations. We then describe different hedging strategies and their impact on the volatility and levels of consumption. We start by showing that in the absence of hedging instruments, Chile should exhibit a significant precautionary business cycle. For example, more than half of the deep reduction in the current account deficit in Chile during the 1998/99 crisis can be accounted for by the optimal response to the fear (not the realization) of a sudden stop.

We then go on to show the benefit of adding contingencies to liability management. For a wide range of scenarios the gains associated with hedging are equivalent to reducing Chile's net external liabilities (of about 50 percent of GDP) by a third. Similarly, we find that the required risk premia to justify for a country like Chile to not adopt an active hedging strategy exceeds 800 basis points for the simplest strategies and several thousand basis points for richer ones. ${ }^{3}$

Of course the benefits of these strategies are not limited to the case of Chile, which is only illustrative. As another example, we show for a panel of emerging markets that the likelihood of sudden stops — as identified by Calvo et al (2005) - exhibits large time variation, and that the latter is strongly correlated with lagged US high yield spreads.

\footnotetext{
${ }^{2}$ We focus on the aggregate financial problem vis-a-vis the rest of the world, in an environment where domestic policy is managed optimally and decentralization is not a source of problems. Needless to say, these assumptions seldom hold in practice. Such failures compound the problems we deal with in this paper by exacerbating the country's exposure to sudden stops. See, e.g., Caballero and Krishnamurthy (2001, 2003) and Tirole (2002) for articles dealing with decentralization problems. The literature on government's excesses is very extensive. See, e.g., Burnside et al (2003) for a recent incarnation.

${ }^{3}$ In his Nobel lecture, Robert Merton (1998) highlights the enormous savings than can be obtained by designing adequate derivatives and other contracting technologies to deal with risk management. He also argues that emergingmarket economies stand to gain the most. Our findings in this paper fully support his views. See also, e.g., Krugman (1988), Froot, Scharfstein, Stein (1989), Haldane (1999), Caballero (2001), for articles advocating commodity indexation of emerging markets debt. The contribution of this paper relative to that literature is to offer a quantitative framework and to link the hedging need not to commodities per se, but to the signal aspect of much costlier financial constraints.
} 
In section 2 we describe the environment and characterize the optimization problem once a sudden stop has been triggered - this provides the boundary conditions for the "precautioning phase." In section 3 we study the phase that precedes a sudden stop when the country self-insures. The main goal of this section is to characterize precautionary contractions. Section 4 describes aggregate hedging strategies under different degree of imperfections in these markets. Section 5 illustrates our results through an application to the case of Chile. Along the way, we outline an econometric approach to gauging the likelihood of a sudden stop and its correlation with an underlying signal. Section 6 provides empirical evidence for a panel of emerging market economies on the large time variation in the likelihood of sudden stops and its correlation with persistent (and exogenous) global indices. Section 7 concludes and is followed by an extensive technical appendix.

\section{Preliminaries}

Intertemporal smoothing implies that emerging economies typically experience substantial needs for external borrowing from abroad. For a variety of reasons that we do not model here, this dependence on borrowing is a source of fragility. The sudden tightening of financial constraints generates large drops in consumption. In this section we formalize such environment and characterize the value function of consumers once a sudden stop has occurred. This serves as a boundary condition for the pre-sudden stop problem faced by the country, which is our main concern in this paper.

\subsection{Endowment and Preferences}

Let the endowment grow at some constant rate, $g>0$, during $0 \leq t<\infty$ :

$$
y_{t}=y_{0} e^{g t}
$$

This specification of the income process excludes feedback effects from aggregate demand to output and from output to sudden stops (although we recover some of the latter channel in the signal process, see below), and hence underestimate the impact of the phenomenon we study. Nonetheless we adopt it because its simplicity allows us to isolate the direct effect of sudden stops more cleanly.

Reflecting emerging markets' net debtor position, the country starts with financial wealth, 
$X(0)=X_{0}<0$. However the country's total wealth $\left(X_{t}+\frac{y_{t}}{r-g}\right)$ must be positive at all times:

$$
X_{t}>-\frac{y_{t}}{r-g} \quad t \geq 0
$$

where $r$ denotes the riskless interest rate and it exceeds the rate of growth of the endowment, $r>g$.

Let $c_{t}$ and $c_{t}^{*}$ represent date $t$ consumption and "excess" consumption, respectively, with:

$$
c_{t}^{*} \equiv c_{t}-\kappa y_{t}, \quad 0 \leq \kappa<1 .
$$

The representative consumer maximizes:

$$
\mathrm{E}_{t}\left[\int_{t}^{\infty} u\left(c_{s}^{*}\right) e^{-\delta(s-t)} d s\right]
$$

with

$$
u\left(c_{t}^{*}\right)=\frac{c^{* 1-\gamma}}{1-\gamma}, \quad \delta>0, \gamma>0
$$

The parameters $\delta$ and $\gamma$ are the discount rate and risk aversion coefficient, respectively. For simplicity, we assume $r=\delta$ throughout. The functional form of the utility function captures an external habit formation, with a habit level that is increasing at the rate of the country's growth rate. The habit term plays two roles: First, it raises the effective risk aversion of the country in a manner similar to Campbell and Cochrane (1999) and thus brings the demand for hedging to realistic magnitudes. Second, it introduces a trend in consumption which allows us to reduce borrowing demand to reasonable levels without introducing overlapping generations or gaps between the interest and discount rates which would complicate the analysis. ${ }^{4}$

\section{A frictionless benchmark}

It is instructive to pause and study the solution of problem (2) subject to a standard intertemporal budget constraint (and absent a sudden stop constraint):

$$
\begin{aligned}
d X_{t}= & \left(r X_{t}-c_{t}^{*}+y_{t}^{*}\right) d t \\
& X_{0} \text { given }
\end{aligned}
$$

\footnotetext{
${ }^{4}$ Accordingly, this type of utility function implies that the indebtedness of the country at any point in time will be larger than $-\frac{(1-\kappa) y_{t}}{r-g}$ :

$$
X_{t}>-\frac{(1-\kappa) y_{t}}{r-g} \quad t \geq 0 .
$$
}


with $y^{*} \equiv(1-\kappa) y$.

By standard reasoning we have that in such case:

$$
c_{t}^{*}=r\left(X_{0}+\frac{y_{0}^{*}}{r-g}\right) \quad \text { for all } t>0,
$$

and the "total resources" of the country remain constant throughout:

$$
X_{t}+\frac{y_{t}^{*}}{r-g}=X_{0}+\frac{y_{0}^{*}}{r-g}
$$

so that

$$
\lim _{t \rightarrow \infty} \frac{X_{t}}{y_{t}^{*}}=-\frac{1}{r-g}
$$

and, accordingly:

$$
\lim _{t \rightarrow \infty} \frac{X_{t}}{y_{t}}=-\frac{1-\kappa}{r-g}
$$

Moreover, for any level of $\frac{X_{t}}{y_{t}}$ above its limit value, the ratio $\frac{X_{t}}{y_{t}^{*}}$ decreases monotonically to $-\frac{1}{r-g}$.

This case serves as a frictionless benchmark in what follows.

\subsubsection{Sudden Stops}

We place no limits on the country's borrowing ability up to the stochastic time $\tau$. At this time, the country faces a "sudden stop." We do not model the informational or contractual factors behind this constraint, or the complex bargaining and restructuring process that follows once the sudden stop is triggered. Since our goal is to produce a quantitative assessment of the hedging aspects of the problem, we look for a realistic and fairly robust (across models) constraint. For this, we simply model the sudden stop as a temporary and severe constraint on the rate of external borrowing. In particular, since the "natural" aggregator of a country's total wealth is the net present value of its total resources, we place the constraint on:

$$
X_{\tau}+\frac{y_{\tau}^{*}}{r-g}
$$

We assume that at time $\tau$, financial markets require the country to increase its total resources by $\zeta$,

$$
e^{g T} \leq \zeta \leq e^{r T}
$$


within $T$ periods. Formally:

$$
\left(X_{\tau+T}+\frac{y_{\tau+T}^{*}}{r-g}\right) \geq \zeta\left(X_{\tau}+\frac{y_{\tau}^{*}}{r-g}\right)
$$

It is apparent that this constraint will be always binding because, as we showed above, at the unconstrained benchmark $\left(X_{t}+\frac{y_{t}^{*}}{r-g}\right)$ remains constant at all times. It is then straightforward to show that this constraint can be expressed as a constraint on the maximum allowable amount of debt/gdp at time $\tau+T$, as a function of the debt/gdp ratio at time $\tau$. To see this, simply rearrange (5) to obtain

$$
\frac{X_{\tau+T}}{y_{\tau+T}^{*}} \geq \zeta e^{-g T} \frac{X_{\tau}}{y_{\tau}^{*}}+\left(\zeta e^{-g T}-1\right) \frac{1}{r-g}
$$

Higher levels of $\zeta$ make the constraint tighter. At its minimum, $\zeta=e^{g T}$, the constraint literally reduces to the requirement that debt/gdp cannot grow any further between $\tau$ and $\tau+T:^{5}$

$$
\frac{X_{\tau+T}}{y_{\tau+T}^{*}} \geq \frac{X_{\tau}}{y_{\tau}^{*}}
$$

Finally, it is interesting to note that one can think of (6) as a constraint on the minimum balance-of-trade surpluses the country has to accumulate over the next $T$ periods. To see this, start with the intertemporal budget constraint:

$$
\int_{\tau}^{\tau+T} e^{-r(t-\tau)}\left(y_{t}^{*}-c_{t}^{*}\right) d t=e^{-r T} X_{\tau+T}-X_{\tau}
$$

and replace (6) into it, to obtain:

$$
\int_{\tau}^{\tau+T} e^{-r(t-\tau)}\left(y_{t}^{*}-c_{t}^{*}\right) d t \geq\left(\zeta e^{-g T}-1\right) \frac{y_{\tau}^{*}}{r-g} e^{-(r-g) T}-\left(1-\zeta e^{-r T}\right) X_{\tau} .
$$

This gives us the constraint in terms of the balance-of-trade surpluses required from a country that is faced with a sudden stop. These required surpluses rise with the country's endowment, with $\zeta$, and with the level of debt at the time of the sudden stop (recall that $X_{\tau}<0$ when the country is a net debtor).

That is, we have arrived to a flow constraint rather than to a conventional stock constraint. This specification not only facilitates our analysis but also captures well policymakers' concerns with current account deficits regardless of the level of external indebtedness. In practice we observe

\footnotetext{
${ }^{5}$ Even for the $\zeta=e^{g T}$ case the constraint we consider is binding at the time of the sudden stop. This follows from the fact that the debt/gdp ratio is always growing for an unconstrained country as we showed above.
} 
a wide array of debt-to-gdp ratios but warnings emerge quickly as soon as current account deficits (a flow) crosses certain thresholds. There is at least one good reason for this concern with flows above and beyond stocks: while there is always some space for stock renegotiation, financing new flows is much harder to obtain during distress.

As we mentioned above. note that while the constraint is on the flows, stocks do matter. A higher debt-to-gdp ratio at the outset of the sudden stop requires a larger consumption sacrifice since debt-servicing is more expensive. This is key for our results below, as the main reason for countries to slow down in anticipation of a sudden stop is to reduce this debt-burden cost. Note also that as $\zeta$ goes to its upper limit $e^{r T}$, the effectiveness of the changes in the initial stock of debt in affecting the constraint is diminished but excess consumption during the sudden stop approaches zero, and hence the value of even small contributions to the relaxation of the constraint have great value. If $\gamma>1$, which we assume throughout, the latter effect dominates and the incentive to adjust debt before a sudden stop rises with $\zeta$.

\subsection{The Sudden Stop Value Function (A Boundary Condition)}

We solve the optimization problem in three steps. Starting backwards, we first solve for the postcrisis period, then for the sudden-stop period, and finally for the period preceding the sudden stop. In this section we summarize the results of the first two steps. The goal of these is to find the value function at the time of the sudden stop, $V^{S S}\left(X_{\tau}, y_{\tau}\right)$, which we then use to find the solution of the optimization and hedging problems before the crisis takes place. Since our concern in this paper is with the latter phase, we assume that there is only one sudden stop, which simplifies the analysis without sacrificing much insight.

Lemma 1 Let $V^{S S}\left(X_{\tau}, y_{\tau}^{*}\right)$ denote the value function at the the time of the sudden stop and set $\gamma>1$. Then:

$$
V^{S S}\left(X_{\tau}, y_{\tau}^{*}\right)=K V^{+}\left(X_{\tau}, y_{\tau}^{*}\right)
$$

where $V^{+}\left(X_{\tau}, y_{\tau}^{*}\right)$ denotes the value function in the benchmark frictionless case:

$$
V^{+}\left(X_{\tau}, y_{\tau}^{*}\right)=\left(\frac{1}{r}\right)^{\gamma} \frac{\left(X_{\tau}+\frac{y_{\tau}^{*}}{r-g}\right)^{1-\gamma}}{1-\gamma}
$$

and the constant $K$ is

$$
K=\left(1-e^{-r T}\right)^{\gamma}\left(1-e^{-r T} \zeta\right)^{1-\gamma}+e^{-r T} \zeta^{1-\gamma},
$$


such that

$$
K=K(\zeta) \quad \text { with } \quad K>1 ; K^{\prime}>0
$$

We leave the proof for the appendix. Note that the value function does not depend on $X_{\tau}$ and

$y_{\tau}$ independently, but instead it depends on the net present value of resources $\left(X_{\tau}+\frac{y_{\tau}^{*}}{r-g}\right)$. This observation will prove convenient later on, because it will allow us to reduce the dimensionality of the dynamic programming problem. Moreover, up to the constant $K$, we have arrived at a value function that is identical to the one in the problem without sudden stops. Lastly, for $\gamma>1$, which we assume throughout, $K>1$ and increases with $\zeta{ }^{6}$

While the particular simplicity of our formulae is due to stylized assumptions, the basic message is more general. The model also can be understood as an approximation to a potentially more complicated specification of constraints that result in higher marginal disutility of debt in the event of a crisis.

\section{Precautionary Contractions}

Let us now address our main concern and begin to characterize the country's problem prior to the sudden-stop phase. If there are no hedging instruments, the country's only mechanism for reducing the cost of a sudden stop is to cut consumption and borrowing before the sudden stop takes place. We show that if sudden stops are somewhat predictable, the amount of self-insurance varies over time. This time varying precautionary savings implies that when the signal of a sudden stop deteriorates, the country falls into a sort of "precautionary contraction." That is, into a sharp reduction in consumption to limit the cost of the potential sudden stop.

Let $s_{t}$ represent a publicly observed sudden stop signal that follows a diffusion process:

$$
d s_{t}=\mu d t+\sigma d B_{t}
$$

\footnotetext{
${ }^{6}$ Observe that for $\gamma>1$ the function $\frac{Z^{1-\gamma}}{1-\gamma}$ is negative for all $Z>0$. Thus $K>1$ reflects that the constrained value function is lower than the continuation value function for the unconstrained problem. The reason we need $\gamma>1$ is that the flow aspect of the constraint implies that having a higher $X_{\tau}$ does not relax the constraint one for one, because the lender does not reduce its demands by the same amount during the sudden stop. When $\gamma$ is below one, this effect is strong enough to discourage saving for the crisis.
} 
This signal is imperfectly related to the sudden stop process. Concretely, the stochastic time $\tau$ depends on the realization of a stochastic jump process, the intensity of which is given by

$$
\lambda_{t}=\exp \left(\alpha_{0}-\alpha_{1} s_{t}\right) ; \quad \alpha_{1} \geq 0 .
$$

We can now write the dynamic programming problem as:

$$
V\left(X_{t}, s_{t}, y_{t}^{*}\right)=\max _{c_{u}^{*}} E\left[\int_{t}^{\tau} \frac{c_{u}^{* 1-\gamma}}{1-\gamma} e^{-r(u-t)} d u+e^{-r(\tau-t)} V^{S S}\left(X_{\tau}, y_{\tau}^{*}\right) \mid F_{t}\right]
$$

where

$$
\tau=\inf \left\{t \in(0, \infty): \int_{0}^{t} \lambda\left(s_{u}\right) d u<z\right\}, \quad \lambda\left(s_{t}\right)=e^{\alpha_{0}-\alpha_{1} s_{t}}, \quad z \sim \exp (1) .
$$

and the evolution of $\left(X_{t}, s_{t}, y_{t}\right)$ is given by:

$$
\begin{aligned}
d X_{t} & =\left(r X_{t}-c_{t}^{*}+y_{t}^{*}\right) d t \\
d y_{t}^{*} & =y_{t}^{*} g d t \\
d s_{t} & =\mu d t+\sigma d B_{t} .
\end{aligned}
$$

From the previous section we know that once the sudden stop takes place, the value function takes the form:

$$
V^{S S}\left(X_{\tau}, y_{\tau}^{*}\right)=K\left(\frac{1}{r}\right)^{\gamma} \frac{\left(X_{\tau}+\frac{y_{\tau}^{*}}{r-g}\right)^{1-\gamma}}{1-\gamma} .
$$

Hence the HJB equation for the value function is:

$$
0=\max _{c^{*}}\left\{\frac{c^{* 1-\gamma}}{1-\gamma}-V_{X} c_{t}^{*}\right\}-r V+V_{X}\left(r X+y^{*}\right)+V_{y^{*}} y^{*} g+V_{s} \mu+\frac{1}{2} \sigma^{2} V_{s s}+\lambda\left(s_{t}\right)\left[V^{S S}-V\right] .
$$

with first order condition:

$$
c_{t}^{*}=\left(V_{X}\right)^{-1 / \gamma} .
$$

In Proposition 5 in the appendix we show that the solution to the HJB takes the simple form:

$$
V=b\left(s_{t}\right)^{\gamma} \frac{\left(X_{t}+\frac{y_{t}^{*}}{r-g}\right)^{1-\gamma}}{1-\gamma}
$$

for an appropriate function $b\left(s_{t}\right)$. The function $b\left(s_{t}\right)$ captures all the economics of the problem and we study it in more detail in what follows. 


\subsection{Precautionary Savings}

Assume first that sudden stops are totally unpredictable, $\alpha_{1}=0$, so that $\lambda_{t}=\exp \left(\alpha_{0}\right)=\lambda$. Since in this case $b(s)$ is no longer a function of the signal $s$, replacing the function $V$ in the HJB equation yields a simple algebraic equation for $b$ :

$$
1-r b+\frac{b}{\gamma} \lambda\left[\left(\frac{b}{b}\right)^{\gamma}-1\right]=0
$$

where

$$
\underline{b} \equiv K^{\frac{1}{\gamma}}\left(\frac{1}{r}\right)
$$

$\underline{b}$ captures the jump in the value function once the country enters into a sudden stop. Let us relegate the proof of the following lemma to the appendix:

Lemma 2 Equation (18) has a unique solution in $\left[\frac{1}{r}, \underline{b}\right]$.

It is now straightforward to obtain the (excess) consumption function from the envelope theorem:

$$
c_{t}^{*}=\frac{\left(X_{t}+\frac{y_{t}^{*}}{r-g}\right)}{b} .
$$

This result -combined with Lemma 2 - demonstrates the presence of precautionary savings: $b$ is

larger than $\frac{1}{r}$, yet smaller than $\underline{b}$. Hence consumption is less than what it would be in the absence of sudden stops. Yet, it is more than what it would be if the country experienced a sudden stop. This reflects the country's desire to accumulate extra savings (compared to the no-sudden stop case) in order to smooth out the effects of these extreme events.

By time-differentiating (19) and using (1), (3), and (18) we obtain the country's (excess) consumption process:

$$
\frac{d c_{t}^{*}}{c_{t}^{*}}=\frac{1}{\gamma} \lambda\left[\left(\frac{\underline{b}}{b}\right)^{\gamma}-1\right] d t>0
$$

That is, the (excess) consumption process prior to a sudden stop has an upward drift: This in turn reflects a precautionary savings demand attributable to the uncertainty generated by the risk of a sudden stop taking place in the next instant. But, because this probability is not correlated with the signal $s$, there are no precautionary contractions. In this case, the pattern of saving for self-insurance is not a source of business cycles. 


\subsection{Time Varying Precautionary Savings}

Let us now return to the general case, where $\alpha_{1}>0$. After a few simplifications, substituting the function $V$ of equation (17) into the HJB equation (15) shows that $b(s)$ satisfies the following ordinary differential equation (ODE):

$$
1-r b+b_{s} \mu+\left[(\gamma-1) \frac{\left(b_{s}\right)^{2}}{b}+b_{s s}\right] \frac{1}{2} \sigma^{2}+\frac{b}{\gamma} \lambda\left(s_{t}\right)\left[\left(\frac{b}{b}\right)^{\gamma}-1\right]=0 .
$$

The boundary conditions reflect the facts that as the signal becomes extremely positive, the problem approximates the frictionless (no sudden stops) benchmark, while when the signal is extremely negative, the problem converges to that at the sudden stop time. Let

$$
\begin{aligned}
b^{*} & =\lim _{s \rightarrow \infty} b(s) . \\
b_{*} & =\lim _{s \rightarrow-\infty} b(s) .
\end{aligned}
$$

where $b^{*}$ and $b_{*}$ are given by:

$$
\begin{aligned}
b^{*} & =\frac{1}{r} \\
b_{*} & =K^{1 / \gamma} \frac{1}{r} .
\end{aligned}
$$

We can easily obtain the solution to the ordinary differential equation (20) numerically. We plot this function (multiplied by $r$ ) in the top subplot of Figure 1. The function $b(s)$ is decreasing with respect to $s$. As the signal deteriorates, this function rises rapidly, reflecting the increasing marginal value of wealth when a sudden stop is likely in the near future.

This setup allows for an explicit characterization of the optimal consumption policy in feedback form. Given the value function, it follows from (16) that the optimal consumption policy takes the form:

$$
c_{t}^{*}=\frac{\left(X_{t}+\frac{y_{t}^{*}}{r-g}\right)}{b\left(s_{t}\right)} .
$$

The effect of the signal can be seen clearly in this expression. As $s$ rises, $b(s)$ falls toward its frictionless limit and hence consumption increases for any given level of wealth. Conversely, when the signal worsens, consumption falls for any given level of wealth causing a precautionary contraction. 

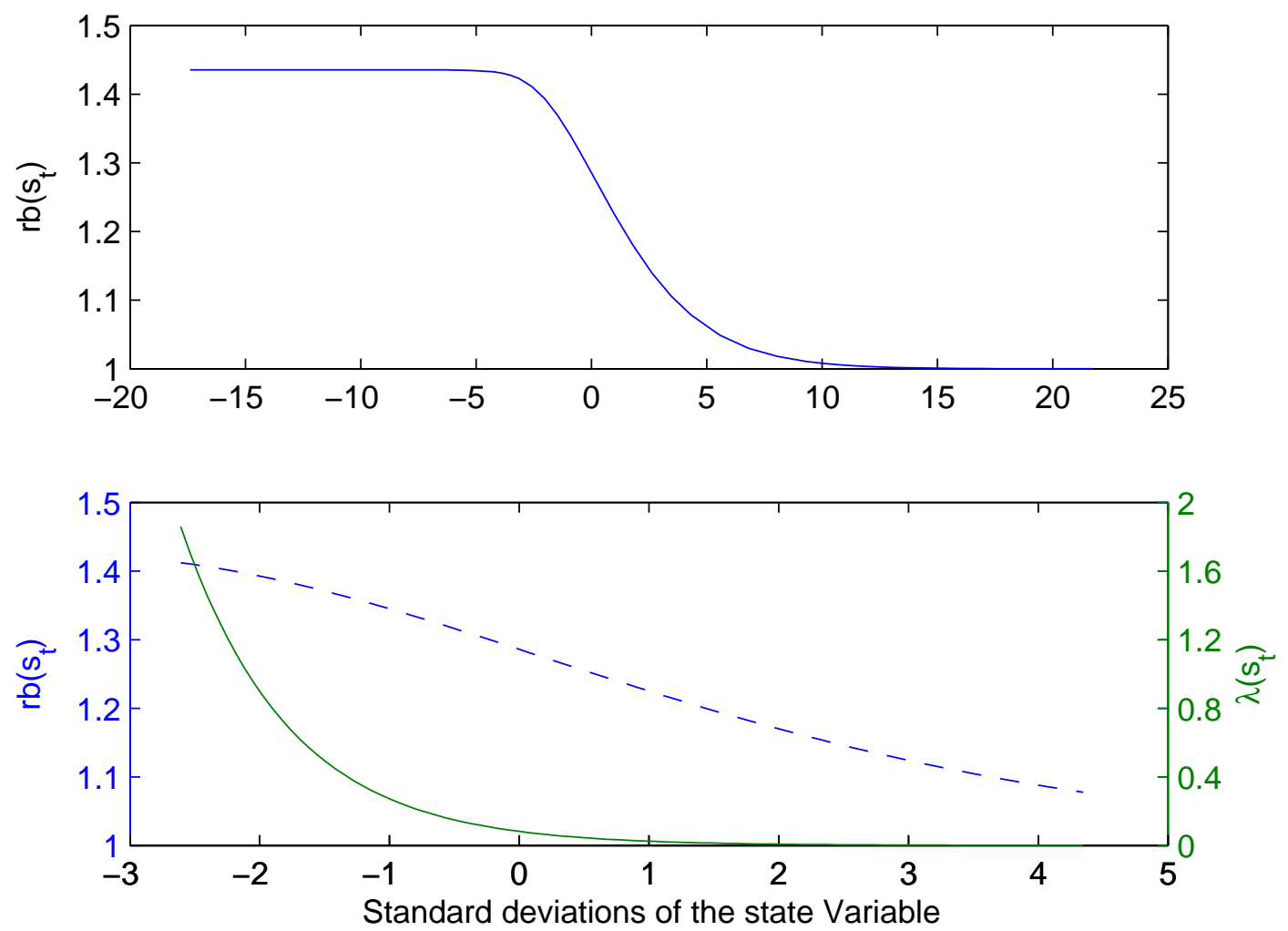

Figure 1: The top subplot depicts $r b\left(s_{t}\right)$ for a wide range of values of $s_{t}$ normalized by its (yearly) standard deviation. Similarly, the bottom panel focuses on a more narrow range of values for $s_{t}$ (normalized by the yearly standard deviation), and plots $r b\left(s_{t}\right)$ (dashed line - measured on the left axis) and the hazard rate (solid line - measured on the right axis). 
Finally, applying Ito's lemma to the right hand side of (23) using (20), (3), and (1) we obtain the country's (excess) consumption process before the sudden stop:

$$
\frac{d c_{t}^{*}}{c_{t}^{*}}=\left[\frac{\gamma+1}{2}\left(\frac{\sigma b_{s}}{b}\right)^{2}+\frac{1}{\gamma} \lambda\left(s_{t}\right)\left[\left(\frac{b}{b}\right)^{\gamma}-1\right]\right] d t-\frac{b_{s}}{b} \sigma d B_{t}
$$

The drift term includes a precautionary term $\left(\frac{1}{\gamma} \lambda\left(s_{t}\right)\left[\left(\frac{b}{\bar{b}}\right)^{\gamma}-1\right]\right)$, which we also found in the case $\alpha_{1}=0$, and captures precautioning against the Poisson jump event that can occur at any time. However, now there are two additional terms associated with the sensitivity of the likelihood of a sudden stop to the signal. For the reasons explained above, $\frac{b_{s}}{b}$ is strictly negative, so that a deteriorating signal increases the need to accumulate resources and accordingly makes consumption respond more to a one-standard-deviation increase in the signal by a factor of $-\frac{b_{s}}{b} \sigma$. There is also an extra precautionary savings term in the drift in response to the additional uncertainty in consumption created by precautionary contractions. In the application section of the paper we quantify these effects.

\subsection{Ignoring time varying hazards}

Before we turn to hedging, we shall develop a framework to measure the costs associated with simply ignoring time-variation in the hazard rate. In order to quantify these costs, assume that a country chose to ignore time variation in the hazard rate and determines its policies as if the hazard rate was given by a constant $\bar{\lambda}$. In that case, the country's "optimal" policy would be given by:

$$
\bar{c}^{*}=\frac{\left(X_{t}+\frac{y_{t}^{*}}{r-g}\right)}{\bar{d}}
$$

where $\bar{d}$ would be a solution to equation (18) with $\lambda$ replacing $\bar{\lambda}$. If the hazard rate is truly time varying, this policy will be clearly suboptimal. We can evaluate the expected utility $V^{s u b}$ associated with this suboptimal policy by plugging it (instead of the optimal policy (23)) into the Bellman equation (15). This results in the partial differential equation:

$$
0=\frac{\left(\bar{c}^{*}\right)^{1-\gamma}}{1-\gamma}-r V^{s u b}+\lambda\left(s_{t}\right)\left(V^{S S}-V^{s u b}\right)+V_{X}^{s u b}\left(r X_{t}+y_{t}^{*}-\bar{c}^{*}\right)+V_{y^{*}}^{s u b} g y_{t}^{*}+V_{s}^{s u b} \mu+\frac{\sigma^{2}}{2} V_{s s}^{s u b}
$$

An informed guess about the solution to this equation is:

$$
V^{s u b}=\left[f\left(s_{t}\right)\right]^{\gamma} \frac{\left(X_{t}+\frac{y_{t}^{*}}{r-g}\right)^{1-\gamma}}{1-\gamma}
$$


Plugging back this conjecture into (25) and simplifying, shows that $V^{s u b}$ solves (25) if and only if $f$ solves:

$$
\begin{aligned}
0= & \frac{1}{\gamma}\left[\left(\frac{f}{\bar{d}}\right)^{1-\gamma}+(\gamma-1) \frac{f}{\bar{d}}\right]-r f+\frac{\lambda\left(s_{t}\right)}{\gamma} f\left(\left(\frac{K^{\frac{1}{\gamma}}}{r f}\right)^{\gamma}-1\right) \\
& +f_{s} \mu+\frac{\sigma^{2}}{2}(\gamma-1) \frac{f_{s}^{2}}{f}+\frac{\sigma^{2}}{2} f_{s s}
\end{aligned}
$$

In the application section we solve this differential equation numerically and evaluate the welfare loses of ignoring time variation in the hazard rate.

\section{Aggregate Hedging}

Precautionary savings and contractions are costly and imperfect self-insurance mechanisms for smoothing the impact of a sudden stop. In this section we enlarge the options of the country and allow it to hedge using insurance contracts and derivatives. Of course, the effectiveness of the hedging strategy depends on the contracts and instruments that are available to the country, and how accurate the crisis-signal is. In this section we provide a framework to explore a variety of scenarios in a unified framework.

\subsection{A unified model of hedging}

There are two fundamental sources of risk in our framework. The first type are risks that are associated with variations in the signal $s$. Since $s$ follows a diffusion process, we shall refer to those as "diffusive" risks. The second source of risks are those that are associated with the actual arrival of the random sudden stop time $\tau$. We shall refer to this type of risk as "jump" risk. The ability of the country to mitigate the effects of sudden stops will critically depend on both the availability of markets to hedge against these risks and the costs of such strategies.

Let us assume that diffusive risks can be hedged in futures market. That is, a country can enter into contracts with payoffs given by:

$$
\frac{d F_{t}}{F_{t}}=\phi_{1} d t+\sigma d B_{t}
$$

where $\phi_{1}$ is a risk premium, that could depend on $s_{t}$. If the diffusive risks are not associated with global risks, then $\phi_{1}=0$ and the futures price is a martingale. In the presence of futures contracts, 
the dynamic budget constraint becomes:

$$
d X_{t}=\left(r X_{t}-c_{t}^{*}+y_{t}^{*}\right) d t+\pi_{t} d F_{t}
$$

where $\pi_{t}$ denotes the number of futures contracts that the country wishes to purchase. Defining the portfolio process as $p_{t}=\pi_{t} F_{t}$, we obtain the new dynamic budget constraint:

$$
d X_{t}=\left(r X_{t}-c_{t}^{*}+y_{t}^{*}+p_{t} \phi_{1}\right) d t+p_{t} \sigma d B_{t}
$$

Let us assume that jump risks are hedged through "insurers" who are willing to enter into an (instantaneous) contract of duration $d t$ in the following terms: If the country enters into a sudden stop over the next interval $d t$, then the insurer pays 1 unit of consumption to the country, else nothing. In exchange for this contract the country pays an amount equal to $\lambda\left(s_{t}\right)\left(1+\phi_{2}\right) d t$ to the insurer per contract purchased. Similar to $\phi_{1}$, the parameter $\phi_{2}$ should be viewed as a markup or a risk premium. If insurance is actuarially fair, then $\phi_{2}=0$. Else the insurer charges $\phi_{2}>0$. Allowing for markets in both diffusive and jump risks, the evolution of assets becomes:

$$
d X_{t}=\left(r X_{t}-c_{t}^{*}+y_{t}^{*}+p_{t} \phi_{1}-\lambda\left(s_{t}\right)\left(1+\phi_{2}\right) I_{t}\right) d t+p_{t} \sigma d B_{t}+I_{t} d N_{t}
$$

where $N_{t}$ is identically equal to 0 and jumps to 1 when the country enters into a sudden stop, and $I_{t}$ denotes the amount of insurance contracts purchased (in units of the numeraire). The dynamic budget constraint (27) includes equations (26) and (3) as special cases. If we assume away the presence of insurers, then $I_{t}=0$ and (27) reduces to (26). Furthermore, if we assume away the presence of futures markets to hedge against diffusive risk, then (26) becomes (3).

\subsection{The general case}

Let us solve the country's problem subject to the dynamic budget constraint (27), and then turn to study important subcases. If the budget constraint is modified to (27), then the Bellman equation for the country becomes:

$$
\begin{aligned}
0= & \max _{c^{*}}\left\{\frac{\left(c^{*}\right)^{1-\gamma}}{1-\gamma}-V_{X} c^{*}\right\} \\
& +\lambda\left(s_{t}\right) \max _{I}\left\{V^{S S}\left(X_{t}+I\right)-V_{X}\left(1+\phi_{2}\right) I\right\} \\
& +\max _{p}\left\{\frac{\sigma^{2}}{2} V_{X X} p^{2}+V_{X s} p \sigma^{2}+p V_{X} \phi_{1}\right\} \\
& +V_{X}\left(r X_{t}+y_{t}^{*}\right)+\mu V_{s}+\frac{\sigma^{2}}{2} V_{s s}+V_{y^{*}} g y_{t}^{*}-\left[r+\lambda\left(s_{t}\right)\right] V
\end{aligned}
$$


The first order conditions for this problem are:

$$
\begin{aligned}
\left(c^{*}\right)^{-\gamma} & =V_{X} \\
V_{X}^{S S} & =V_{X}\left(1+\phi_{2}\right) \\
p & =-\frac{V_{X}}{V_{X X}} \frac{\phi_{1}}{\sigma^{2}}-\frac{V_{X s}}{V_{X X}}
\end{aligned}
$$

The first optimality condition is the familiar Euler equation. The second equation captures the country's attempt to equate the marginal value of a dollar pre- and post- sudden stop, which is fully accomplished if $\phi_{2}=0$. Finally, the last condition is identical to Merton's formula. The optimal portfolio of futures consists of two parts: The first part is positive (since $V_{X X}<0, V_{X}>0$ ) and captures the willingness to accept some volatility in return for a risk-premium. By contrast, a simple intuitive argument shows that the second term captures a hedging demand and is negative. The reason is that the marginal value of wealth will become higher the more likely it becomes that a sudden stop is imminent. This happens as $s$ decreases and hence $V_{X s}<0$. A particularly interesting case is $\phi_{1}=0$, i.e. when the signal is uncorrelated with the world economy. Then $p<0$, since only the hedging motive prevails.

To solve (28) we proceed as in Section 3 and conjecture that the value function has a separable form:

$$
V=d\left(s_{t}\right)^{\gamma} \frac{\left(X_{t}+\frac{y_{t}^{*}}{r-g}\right)^{1-\gamma}}{1-\gamma}
$$

With this conjecture, equations (29) through (31) become, respectively:

$$
\begin{aligned}
c_{t}^{*} & =\frac{1}{d\left(s_{t}\right)}\left(X_{t}+\frac{y_{t}^{*}}{r-g}\right) \\
I & =\left[\frac{1}{r d}\left(\frac{\left(1+\phi_{2}\right)}{K}\right)^{-\frac{1}{\gamma}}-1\right]\left(X_{t}+\frac{y_{t}^{*}}{r-g}\right) \\
p & =\left(\frac{1}{\gamma} \frac{\phi_{1}}{\sigma^{2}}+\frac{d_{s}}{d}\right)\left(X_{t}+\frac{y_{t}^{*}}{r-g}\right)
\end{aligned}
$$

It will be useful to define:

$$
i=\frac{I}{\left(X_{t}+\frac{y_{t}^{*}}{r-g}\right)}
$$

Plugging back (32) into (28), using the above four equations and performing several simplifications, 
shows that $V$ satisfies (28) if and only if $d\left(s_{t}\right)$ satisfies the equation:

$$
\begin{aligned}
0= & 1-r d+\mu d_{s}+\frac{\sigma^{2}}{2} d_{s s}+ \\
& +\frac{1-\gamma}{\gamma}\left[d \frac{\gamma \sigma^{2}}{2}\left(\frac{1}{\gamma} \frac{\phi_{1}}{\sigma^{2}}+\frac{d_{s}}{d}\right)^{2}-\frac{\gamma \sigma^{2}}{2} \frac{\left(d_{s}\right)^{2}}{d}\right] \\
& +\frac{1-\gamma}{\gamma} \lambda\left(s_{t}\right)\left\{\left(\frac{\gamma}{1-\gamma}\right)\left(\frac{K^{\frac{1}{\gamma}}}{r}\right)\left(1+\phi_{2}\right)^{1-\frac{1}{\gamma}}+\left(1+\phi_{2}\right) d-\frac{d}{1-\gamma}\right\}
\end{aligned}
$$

Equation (36) is a non-linear second order differential equation which is to be solved under the boundary conditions (21) and (22). Even though it does not have an analytical solution, we can still characterize several properties of the solution. Of particular interest is the behavior of consumption, for which we have:

Lemma 3 The excess consumption process evolves according to:

$$
\begin{aligned}
\frac{d c^{*}}{c^{*}}= & -\frac{1}{d}\left[1-r d+\lambda\left(s_{t}\right)\left(1+\phi_{2}\right) i d+\left(\mu+\frac{\phi_{1}}{\gamma}\right) d_{s}+\frac{\sigma^{2}}{2} d_{s s}\right] d t \\
& +\left(\frac{1}{\gamma} \frac{\phi_{1}}{\sigma}\right) d B_{t}
\end{aligned}
$$

Let us now analyze a few special cases to understand the different component of the consumption process.

\subsection{No restrictions on hedging}

The natural starting point is when $\phi_{1}=\phi_{2}=0$. In other words, markets exist for both types of risks and the risks that threaten the country are diversifiable. The following Lemma shows that in this case pre-sudden stop consumption will be constant:

Proposition 1 If $\phi_{1}=\phi_{2}=0$, then

$$
d c_{t}^{*}=0, \quad 0 \leq t<\tau
$$

This result asserts that consumption will be constant prior to a sudden stop if a country can hedge perfectly in markets for both diffusive and jump risks. This result is hardly surprising. If the

country can costlessly trade in contingent claims, then it will be able to stabilize its consumption stream pre-sudden stop. Precautionary savings ceases to be necessary as a mitigation mechanism. 
Let us pause and note that both markets need to be costelessly accessible in order to obtain the above result. This may seem surprising at first. Why should there be a need for a market for diffusive risks as long as the country can costelessly contract with insurers on the amounts it desires upon the arrival of the sudden stop? After all, variations in $s$ are only useful in predicting the likelihood of a jump, and are otherwise useless. The resolution of this puzzle is that the cost of insurance is time varying. Since the contracts with the insurers are instantaneous, there is uncertainty about the terms under which these contracts can be rolled over in the future. If $s$ decreases, then the actuarially fair insurance will become more costly. Therefore, futures are needed in order to hedge against such variations in the price of insurance.

To see this, let us momentarily set $\phi_{2}=0$, but

$$
\phi_{1}=-\gamma \sigma^{2} \frac{d_{s}}{d}
$$

In the appendix we show that in this case the (excess) consumption process has the following properties:

Proposition 2 Assume that $\phi_{2}=0$ and $\phi_{1}=-\gamma \sigma^{2} \frac{d_{s}}{d}$. Then

$$
\begin{aligned}
\frac{d c^{*}}{c^{*}}= & \frac{1}{d}\left[(\gamma+1) \frac{\sigma^{2}}{2} \frac{\left(d_{s}\right)^{2}}{d}\right] d t \\
& -\frac{d_{s}}{d} \sigma d B_{t}
\end{aligned}
$$

for $d\left(s_{t}\right)$ that solves (36) with $\phi_{2}=0$ and $\phi_{1}=-\gamma \sigma^{2} \frac{d_{s}}{d}$.

That is, the consumption process is a non-degenarate diffusion (in the sense that it has a nonzero " $d B_{t}^{\prime \prime}$ term). This shows that in the presence of time variation in the likelihood of a sudden stop both markets (for diffusive and jump risk) will contribute towards hedging. Neither will be sufficient in isolation.

Let us not return to the case with $\phi_{1}=\phi_{2}=0$. An alternative way to arrive at Proposition 1 is to use the martingale methods developed by Cox and Huang (1989) and Karatzas et.al. (1987). This methodology is particularly attractive when dynamic trading allows one to span all possible stochastic payoffs. 
Formally, these methods start by solving an inherently static problem. Namely:

$$
\max _{c_{u}^{*}, X_{\tau}} E\left[\int_{t}^{\tau} \frac{c_{u}^{* 1-\gamma}}{1-\gamma} e^{-r(u-t)} d u+e^{-r(\tau-t)} V^{S S}\left(X_{\tau}, y_{\tau}^{*}\right) \mid F_{t}\right]
$$

subject to the intertemporal budget constraint:

$$
E\left[\int_{t}^{\tau} e^{-r(u-t)} c_{u}^{*} d u\right]+E\left(e^{-r(\tau-t)} X_{\tau}\right) \leq X_{t}+E\left[\int_{t}^{\tau} e^{-r(u-t)} y_{u}^{*} d u\right]
$$

This is an inherently static problem. Adjoining a lagrange multiplier $k$ to the budget constraint (39) and maximizing (38) over $c_{u}^{*}, X_{\tau}$ gives:

$$
\begin{aligned}
c_{u}^{*} & =k^{-\frac{1}{\gamma}}, \quad t \leq u \leq \tau \\
X_{\tau} & =\frac{1}{r}\left(\frac{k}{K}\right)^{-\frac{1}{\gamma}}-\frac{y_{\tau}^{*}}{r-g}
\end{aligned}
$$

There are at least two observations that deserve comment. First, observe that the (excess) consumption process is equal to a constant for all times between $t \leq u \leq \tau$. This is just a restatement of Proposition 1. Second, observe that the optimal level of financial wealth at time $\tau$ can be decomposed into two components:

$$
X_{\tau}=\underbrace{\left[\frac{1}{r} k^{-\frac{1}{\gamma}}-\frac{y_{\tau}^{*}}{r-g}\right]}_{\text {Optimal Wealth in the absence of SS }}+\underbrace{\frac{1}{r} k^{-\frac{1}{\gamma}}\left[(K)^{\frac{1}{\gamma}}-1\right]}_{\text {Extra insurance needed to cover SS }}
$$

Since $K>1$ the second term is clearly positive and it captures the demand for insurance. Combining (39), (40) and (41) we can solve for $c_{u}^{*}=k^{-\frac{1}{\gamma}}$

$$
c_{t}^{*}=k^{-\frac{1}{\gamma}}=\frac{r\left(X_{t}+\frac{y_{t}^{*}}{r-g}\right)}{1+\left(K^{\frac{1}{\gamma}}-1\right) E e^{-r(\tau-t)}}
$$

This formula shows that optimal (excess) consumption will be lower in an economy that is exposed to sudden stops compared to an economy that is not exposed to such events by the factor: ${ }^{7}$

$$
\frac{1}{1+\left(K^{\frac{1}{\gamma}}-1\right) E e^{-r(\tau-t)}}
$$

\footnotetext{
${ }^{7}$ It is interesting to cross-check that dynamic programming and the martingale methods produce the same answer for the consumption process. To see this note that the martingale methods in conjunction with $(33)$ imply that $d\left(s_{t}\right)$ is given by :$$
d\left(s_{t}\right)=\frac{1}{r}+\frac{1}{r}\left(K^{\frac{1}{\gamma}}-1\right) E e^{-r(\tau-t)}
$$ 
The reason is intuitive: Even when all hedging possibilities are unconstrained, the country still has to pay for such insurance, and hence it will have to cut back consumption somewhat in order to be able to finance this insurance. However, this insurance will allow the country to eliminate the volatility of its consumption process subsequently. An interesting feature of (42) is that it allows us to determine the cost of "hedging" as a percentage of excess consumption. That cost is given as

$$
\frac{r\left(X_{t}+\frac{y_{t}^{*}}{r-g}\right)-c_{t}^{*}}{c_{t}^{*}}=\frac{1-\frac{1}{1+\left(K^{\frac{1}{\gamma}}-1\right) E e^{-r(\tau-t)}}}{c_{t}^{*}}=\left(K^{\frac{1}{\gamma}}-1\right) E e^{-r(\tau-t)}
$$

This formula allows a simple "back of the envelope" computation. Note that $E e^{-r(\tau-t)}$ is the moment generating function of the random time $\tau$. Accordingly:

$$
E e^{-r(\tau-t)}=1-r E(\tau-t)+O\left(r^{2}\right)
$$

Ignoring the last term in the above equation, the cost of insurance as a percentage of excess consumption is given approximately as:

$$
\left(K^{\frac{1}{\gamma}}-1\right)(1-r E(\tau-t))
$$

This formula is quite intuitive. It says that the cost of hedging is proportional to the severity of the sudden stop (as captured by $K^{1 / \gamma}$ ) and the constant of proportionality is given by $(1-r E(\tau-t))$. Hence, the longer is the expected distance to the sudden stop (say because $s$ is high) the less is the cost of insurance.

To see that dynamic programming will produce the same answer, note that according to the Bellman equation (36) and the fact that $\phi_{1}=\phi_{2}=0$ :

$$
0=1-r d+\mu d_{s}+\frac{\sigma^{2}}{2} d_{s s}+\lambda\left(s_{t}\right)\left\{\left(\frac{K^{\frac{1}{\gamma}}}{r}\right)-d\right\}
$$

According to the Feynman Kac Theorem the solution to this linear ODE is given by:

$$
d\left(s_{t}\right)=E \int_{t}^{\tau} e^{-r(s-t)} d s+E e^{-r(\tau-t)}\left(\frac{K^{\frac{1}{\gamma}}}{r}\right)
$$

which in turn is equal to:

$$
d\left(s_{t}\right)=E\left(\int_{t}^{\tau} e^{-r(s-t)} d s+\int_{\tau}^{\infty} e^{-r(s-t)} d s\right)+E\left[e^{-r(\tau-t)}\left(\frac{K^{\frac{1}{\gamma}}}{r}-\int_{\tau}^{\infty} e^{-r(s-\tau)} d s\right)\right]
$$

and therefore

$$
d\left(s_{t}\right)=\frac{1}{r}+E\left[e^{-r(\tau-t)}\left(\frac{K^{\frac{1}{\gamma}}}{r}-\frac{1}{r}\right)\right]
$$

This is exactly the same expression as the one produced by the martingale approach. 


\subsection{Restricted insurance}

One could reasonably argue that the hedging of jump risks may be infeasible in practice. Insuring sudden stops directly probably requires to overcome a long list of moral hazard and informational concerns. One possibility is to do proxy-hedging using exogenous variables, as described by Caballero and Panageas (2005), but there will always be an important residual that probably can only be insured (and to a limited amount) by international financial institutions rather than by private markets.

However, if one can find tradeable variables (such as the price of copper) that are useful in predicting the arrival of a sudden stop, then hedging "diffusive" risk alone can still provide significant benefits.

The easiest way to see this is to impose the constraint $I=0$, but continue to maintain the assumption that $\phi_{1}=0$. Let us enforce the constraint $I=0$ by assuming that $\phi_{2}$ satisfies:

$$
\phi_{2}=\frac{K}{(r d)^{\gamma}}-1
$$

If $\phi_{2}$ is given by this process, then clearly $I=0$ by (34). It is most useful to think of $\phi_{2}$ not as a risk premium but as the Lagrange multiplier associated with the constraint $I=0$. The following result shows that even in this case the consumption process is less volatile than in Section 3.

Proposition 3 Assume that $\phi_{2}$ is given by (44) so that $I=0$. Furthermore assume that $\phi_{1}=0$. Then the optimal consumption process is given by:

$$
\frac{d c^{*}}{c^{*}}=\frac{1}{\gamma} \lambda\left(s_{t}\right)\left\{\left(\frac{K^{1 / \gamma}}{r d}\right)^{\gamma}-1\right\} d t
$$

where $d$ solves (36) with $\phi_{2}$ given by (44) and $\phi_{1}=0$.

A key observation about the above proposition is that the consumption process has no quadratic variation. This is in contrast to Section 3 where we allowed precautionary savings only (compare with equation (37)). This means that hedging with futures achieves the goal of mitigating precautionary contractions, even in the complete absence of markets that insure against the arrival of sudden stops (jump risk).

Note, however, that relative to the $\phi_{2}=0$ case, the consumption process still has a drift reflecting the presence of precautionary savings due to the presence of unhedged jump risk. Moreover, 
there is still (smooth) variation in the consumption rate pre-sudden stop since both $\lambda$ and $d$ depend on $s_{t}$.

\subsection{Pure Precautionary savings as a special case}

In the previous section we postulated equation (44) for $\phi_{2}$ in order to impose the constraint $I=0$. In this section we shall additionally postulate that $p=0$, so that the country is excluded from hedging possibilities altogether. From equation (35) we set $p=0$ by setting:

$$
\phi_{1}=-\gamma \sigma^{2} \frac{d_{s}}{d}
$$

That is, $\phi_{1}$ is the appropriate "shadow" risk premium that ensures that $p=0$ throughout. The next proposition shows that under the joint assumptions (44) and (45) we recover the result in section 3.2 .

Proposition 4 Assume that (44) and (45) hold. Then $d\left(s_{t}\right)$ solves the same equation as $b\left(s_{t}\right)$ in section 3.2 and the consumption process is given by

$$
\begin{aligned}
\frac{d c^{*}}{c^{*}}= & \frac{1}{d}\left[\frac{1}{\gamma} \lambda\left(s_{t}\right) d\left\{\left(\frac{K^{1 / \gamma}}{r d}\right)^{\gamma}-1\right\}+(\gamma+1) \frac{\sigma^{2}}{2} \frac{\left(d_{s}\right)^{2}}{d}\right] d t \\
& -\frac{d_{s}}{d} \sigma d B_{t}
\end{aligned}
$$

which is identical to equation (24).

The value of this proposition is to embed the results in Section 3 in the same framework as the one that we used in this section and, more importantly, to identify the necessary assumptions on "shadow" risk premia in order to justify no hedging.

\subsection{Discussion}

We can now view the results that we have obtained thus far in a unified framework. In Section 3 we eliminated every hedging possibility. Therefore, the country was left with precautionary savings as its sole protection against sudden stops. The country's consumption pre-sudden stop was affected by both variations in the hazard rate (diffusive risk) and the random arrival of the sudden stop conditional on the hazard rate (jump risk). At the polar opposite, Section 4.3 allowed for the 
presence of an insurer and a market to hedge against diffusive risk. This helped eliminate the effects of both sources of risk on consumption. Hence consumption pre-sudden stop was constant. Subsection 4.4 helped demonstrate that even in the absence of an insurer, the presence of hedging markets can help eliminate the effects of diffusive risk on consumption. However, the consumption process will still be affected by the non-insurability of jump risk which leaves a random drift in the consumption process.

Importantly, Sections 4.4 and 4.5 helped to demonstrate that the assumptions on the presence or absence of markets are equivalent to making appropriate assumptions on the prevailing "shadow" risk premia on these markets. This will facilitate robustness checks after we calculate optimal hedging strategies.

\section{An Illustration: The Case of Chile}

In this section we illustrate our main results through an application to the case of Chile. This is a good case study since Chile is an open economy, with most of its recent business cycle attributable to capital flows' volatility. Moreover, the price of copper (its main export) is not only an important source of income fluctuations but, more importantly for us, it is an excellent indicator of investors attitude toward Chile. In fact, Caballero (2001) shows that the typical contraction in Chilean aggregate demand and net capital flows after a sharp decline in the price of copper is many times larger than the corresponding annuity value of the decline in Chilean wealth due to the drop in copper prices.

\subsection{Calibration}

While our purpose here is only illustrative, and hence our search for parameters is rather informal, we spend some time describing our estimation/calibration of the key function $\lambda(s)$.

\subsubsection{Estimating $\lambda(s)$}

Sudden stops are severe but rare events, which makes it hard to estimate $\lambda(s)$ with any precision. However, we still highlight our procedure because it provides a good starting point for an actual implementation. Similarly, a key issue is determining the components of the signal, $s$. Given 
the limited goal of this section, we use only the logarithm of the price of copper. In a true implementation it also would be worth including some global risk-financial indicator, such as the U.S. high yield spread, the EMBI+ or the VIX (see Section 6 below), and removing slow moving trends from the price of copper. ${ }^{8}$

With these caveats behind us, let us describe the reduced-form Markov regime switching model we use to estimate $\lambda(s)$. Unlike the standard regime-switching model, ours has time-varying transition probabilities. Our left-hand side variable is aggregate demand, $Y_{t}$ (which we will proxy with GDP data but it does not make any difference for our purpose of identifying the likelihood of a sudden stop at each point in time) and is generated by the process:

$$
Y_{t}=\mu_{0}+1_{\left\{z_{t}=1\right\}} \mu_{1}+\sigma_{z_{t}} \varepsilon, \quad \varepsilon \sim N(0,1), \mu_{1}<0
$$

The growth rate of $Y_{t}$ depends on an unobserved regime $z_{t}$ that takes values 0 and 1 . When the value of the regime is 0 , the regime is "normal" and growth is just a normal variable with mean $\mu_{0}$ and variance $\sigma_{0}^{2}$. Otherwise, the regime is "abnormal" and growth has a lower mean, $\mu_{0}+\mu_{1}$, and a variance $\sigma_{1}^{2}$. The transition matrix between the two states is assumed to be the following:

$$
\begin{array}{cc}
\operatorname{Pr}\left(z_{t+1}=0 \mid z_{t}=0, X_{s, s \in(t, t+1)}\right)=\exp ^{-\int_{t}^{t+1} \lambda\left(x_{u}\right) d u}=p_{00} & \operatorname{Pr}\left(z_{t+1}=1 \mid z_{t}=0, X_{s, s \in(t, t+1)}\right)=p_{01} \\
\operatorname{Pr}\left(z_{t+1}=0 \mid z_{t}=1, X_{s, s \in(t, t+1)}\right)=p_{10} & \operatorname{Pr}\left(z_{t+1}=1 \mid z_{t}=1, X_{s, s \in(t, t+1)}\right)=q
\end{array}
$$

where $p_{01}=1-p_{00}, p_{10}=1-q$, and $X_{t}$ stands for the regressors that enter the hazard function (the logarithm of the price of copper in this application). ${ }^{9}$ For the purpose of estimating $\lambda(s)$, we use annual GDP data starting from 1976 to 1999 and monthly copper data for the same period (source, International Financial Statistics). ${ }^{10}$

\footnotetext{
${ }^{8}$ Removing the slow moving trend not only seems to improve the fit but also is a key ingredient in designing long run insurance and hedging contracts. Few investors/insurers are likely to be willing to hold long-term risk on a variable that may turn out to be non-stationary.

${ }^{9}$ Strictly speaking the quantity $\exp ^{-\int_{t}^{t+1} \lambda\left(x_{u}\right) d u}$ is unobservable, since we cannot observe the continuous path of copper, only discrete points. However, we can obtain copper data at reasonable high frequencies so that we can safely ignore this issue and calculate the integral by a Rieman sum as

$$
\exp ^{-\int_{t}^{t+1} \lambda\left(x_{u}\right) d u} \approx \exp ^{-\sum \lambda\left(x_{u}\right) \Delta u}
$$

${ }^{10}$ There is a caveat here. Our extended-sample starts from 1972, but all the years up to 1976 are part of a deep
} 
For estimation, we use a Bayesian approach that is suitable for the very few datapoints that we have. Bayesian inference seems natural in this context, since it allows exact statements that do not require asymptotic justification. To estimate the parameters of interest (namely $\left(\alpha_{0}, \alpha_{1}\right)$ ) we use a multimove Gibbs Sampler as described in Kim and Nelson $(1998,1999)$ and is based on the filtering algorithm of Hamilton (1989). For details we refer the reader to these references. The basic idea is to augment the parameter set by treating the unobserved states as parameters. Then we fix a draw from the posterior distribution of $\left(\mu_{0}, \mu_{1}, \sigma_{0}, \sigma_{1}, q, \alpha_{0}, \alpha_{1}\right)$ and conditional on these parameters we draw from the posterior distribution of the states in a single step as described in Kim and Nelson (1999). Given the draw from the posterior distribution of the states we sample the conditional means $\mu_{0}, \mu_{1}$ using a conjugate normal / truncated normal prior which leads to a normal / truncated normal posterior. ${ }^{11}$ Conditional on the states and the draw from $\left(\mu_{0}, \mu_{1}\right)$ se sample from the posterior distribution of $\left(\sigma_{0}, \sigma_{1}\right)$ using inverse gamma priors which lead to posteriors in the same class. Similarly, fixing the states and the draw from $\left(\mu_{0}, \mu_{1}, \sigma_{0}, \sigma_{1}\right)$ we draw $q$ using a conjugate beta prior leading to conjugate beta posterior. The sampling of $\left(\alpha_{0}, \alpha_{1}\right)$ presents the difficulty that there does not seem to be a natural conjugate prior to use and thus we use a Metropolis Hastings-Random walk-accept-reject step where we sample from a bivariate normal centered at the previous iterations' estimate as described in Robert and Casella (1999). This provides us with a new draw form $\left(\mu_{0}, \mu_{1}, \sigma_{0}, \sigma_{1}, q, \alpha_{0}, \alpha_{1}\right)$ and conditional on this new draw we can iterate the algorithm by filtering the states again, based on the new draw etc. It is then a standard result in Bayesian computation that the stationary distribution of the parameters sampled with these procedure (treating the unobserved states as parameters too) coincides with the posterior distribution of the parameters. ${ }^{12}$

contraction due to political turmoil combined with extremely weak external conditions. However, since the extendedsample starts during abnormal years these do not influence the estimation of $\lambda(s)$ (which is estimated from the transitions from normal to abnormal states). It is in this sense that the sample relevant for the estimation of the latter starts in 1976. For details see below.

${ }^{11}$ For details see Albert and Chib (1993).

${ }^{12}$ To reduce computational time and satisfy the technical conditions required for the applicability of the Gibbs Sampler, we used proper priors for $\left(\mu_{0}, \mu_{1}\right)$ and $\left(\alpha_{0}, \alpha_{1}\right)$ and improper priors for the rest of the parameters. The proper prior for $\left(\mu_{0}, \mu_{1}\right)$ was Normal / Truncated normal with means $(0,0)$ and a diagonal covariance matrix. The standard deviations where chosen to be roughly 5 times the range of the sample, so that the priors had effectively no influence on the estimation. Similarly for $\left(\alpha_{0}, \alpha_{1}\right)$ we used independent normal priors centered at 0 with a standard 
A first output of this procedure is Figure 2 which plots the probability of being in an abnormal state. The model recognizes roughly three years out of the 24 as abnormal years: the early 1980s and the recent episode following the Asian/Russian crises. The early 1980s episode corresponds to a devastating debt-crisis, and was significantly more severe than the recent one. In fact, the recent episode appears to be a mix of a milder sudden stop and a precautionary recession.

An interesting observation about these probabilities is that they allow us to identify the abnormal regimes with great confidence. To improve the tightness of the posterior confidence intervals concerning the parameters of interest $\left(\alpha_{0}, \alpha_{1}\right)$ we observe that conditional on the states the $(\log -)$ likelihood function becomes:

$$
\sum_{z_{i}=0}\left[\chi_{i} \log \left(p_{00}\left(\alpha_{0}, \alpha_{1}\right)\right)+\left(1-\chi_{i}\right) \log \left(p_{01}\left(\alpha_{0}, \alpha_{1}\right)\right)\right]
$$

where $\chi_{i}$ takes the value 1 if there is no transition to state 1 , and takes the value 0 otherwise. It is interesting to notice that the likelihood for $\left(\alpha_{0}, \alpha_{1}\right)$ depends on the data only through the filtered states. In other words all other parameters of the model including the GDP-data are relevant for our purpose of estimating $\left(\alpha_{0}, \alpha_{1}\right)$ only to the extent that they influence the identification of the states. In other words if we were to condition directly on the states we would be able to get rid of the noise introduced by filtering.

Given the few data points and the quite clear identification of the abnormal states we report directly the posterior distribution of $\left(\alpha_{0}, \alpha_{1}\right)$ conditional on the early 80's and 1999 being the abnormal states. we report the posterior distributions of the parameters $\left(\alpha_{0}, \alpha_{1}\right)$ conditional on the states that are identified as abnormal. ${ }^{13}$ Table 1 reports these results.

Correspondingly, in what follows we use $\alpha_{0}=2.5, \alpha_{1}=5.2$ as our benchmark values for the function $\lambda(s)$.

deviation of 10. Even when we experimented with more diffuse priors the algorithm produced virtually identical results but computational time was significantly increased, because convergence was significantly slower. Most importantly though, for the results that we report in Table 1 and that we use in the calibration exercise we used completely flat priors.

${ }^{13}$ I.e., states that have a posterior probability of being abnormal above 0.75 . The conditioning is done in order to increase the precision of the estimates and seems to be warranted just by a visual inspection of Figure 2 . 


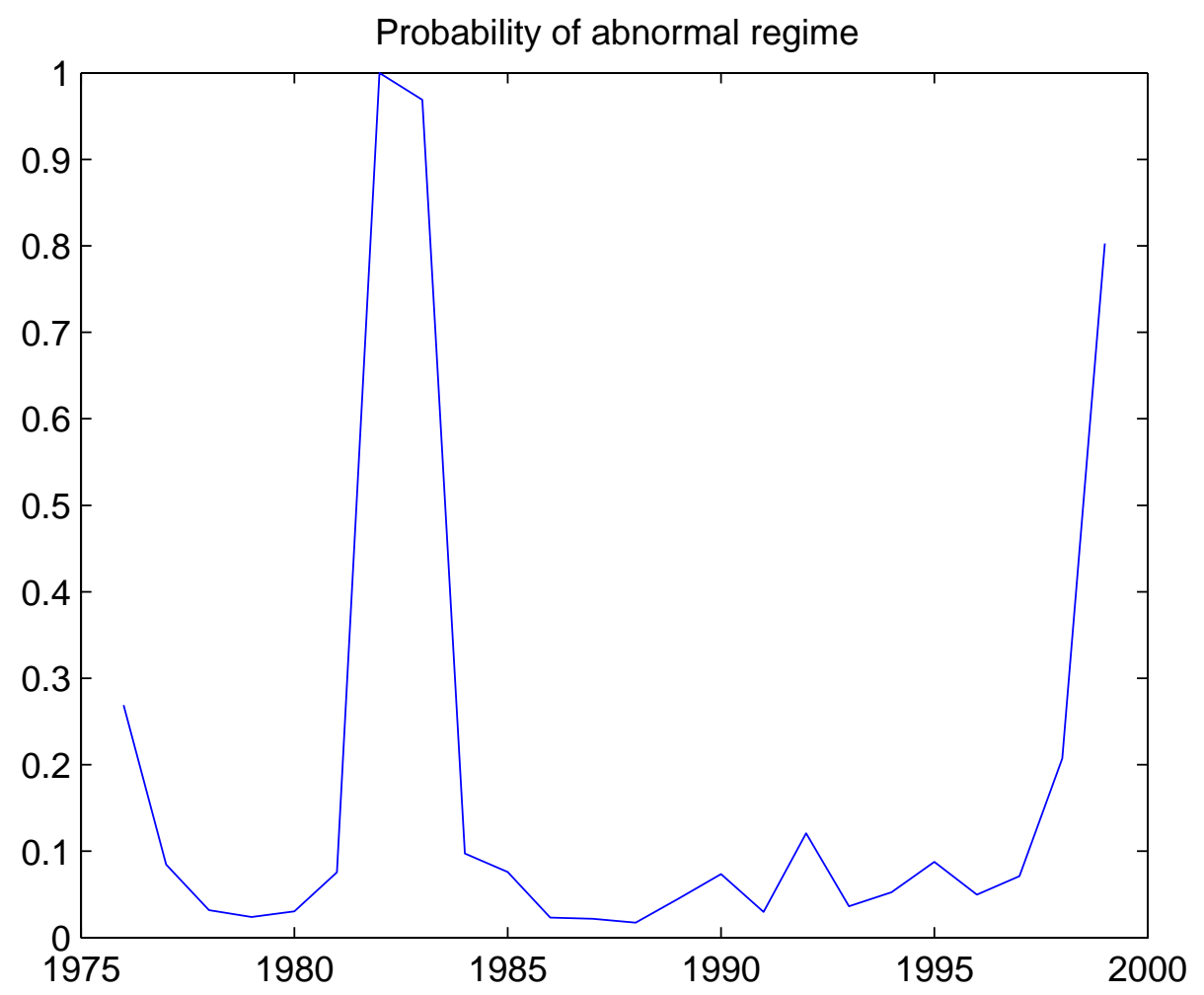

Figure 2: Probability of abnormal regime

\begin{tabular}{lrrrrrrr}
\hline Posterior Distributions & \multicolumn{1}{c}{} & & & & & \\
\hline & mean & std. Deviation & 10 & 25 & 50 & 75 & 90 \\
\hline$\alpha_{0}$ & -2.522 & 1.067 & -3.826 & -3.027 & -2.316 & -1.789 & -1.420 \\
$\alpha_{1}$ & -5.170 & 3.387 & -9.259 & -6.802 & -4.708 & -3.040 & -1.515 \\
\hline
\end{tabular}

Table 1: Posterior Distributions of the Parameters $\left(\alpha_{0}, \alpha_{1}\right)$ Conditional on the States that are Identified as Abnormal. 10, 25 etc. refer to the respective quantiles. 


\subsubsection{Other parameters}

We calibrate $\zeta$ and $T$ to generate the average cumulative consumption drop caused by the sudden stops in the sample, which is about $8-10 \%$ of GDP. One such configuration is $T=1$ and $\zeta=$ $e^{g T}+0.35\left(e^{r T}-e^{g T}\right)$.

The parameters $\kappa$ and $\gamma$ are calibrated to generate reasonable amounts of steady-state debt (and hence reasonable amounts of insurance needs) together with significant precautionary fluctuations (see below, in particular, to explain much — but not all - of the precautionary contraction experienced by Chile at the early stages of the 1998-9 episode). For this, we set $\kappa=0.8$ and $\gamma=7$.

The interest rate $r$ is set to 0.09 to capture the high cost of borrowing faced by the typical emerging market and the growth rate $g$ is set to 0.03 . The latter is a constant-rate approximation to a path that grows significantly faster than that level for a few years, while the country is catching up, and then decelerates below that level forever. We normalize initial GDP $\left(y_{0}\right)$ to 1 , and set the initial debt-to-GDP ratio to 0.5 (i.e., $X_{0}=-0.5$ ).

Finally, we approximate the process for copper by a driftless Brownian motion with constant volatility $\sigma$, which we estimate using monthly data from 1972 to 1999 (source, IFS). We find a value of roughly $\sigma=0.23$ and normalize the initial value of $s$ to 0 .

\subsection{Aggregate Hedging}

Given these parameters, we now turn to a quantitative evaluation of the effectiveness of the various strategies described in Sections 3.2 and 4.

\subsubsection{Precautionary Savings and Hedging}

Let us illustrate the impact of the different hedging strategies in steps: In the first one we illustrate the gains from going from a pure precautionary savings strategy to one that incorporates diffusion risk hedging. In the second one we enrich the strategy further by adding jump risk hedging.

We start by drawing a random path for $s_{t}$, plotted in panel (a) of Figure 3. The path runs for 8 years and no sudden stop takes place in it. The main features of this path are not too different from the realization of the price of copper during the 1990s. In particular, the large rise in $s$ near the middle of the path followed by a sharp decline toward the end of the period is reminiscent of 
the path of the price of copper in the second half of the 1990s. The path starts at $s_{0}=0$, which corresponds to the empirical mean of $s_{t}$ in the data.

The two lines in subplot (b) illustrate the corresponding paths of (excess) consumption generated for the precautionary savings only case (dashed line) and the diffusive risk hedging case (solid line). The former tracks the signal closely while the latter manages to avoid precautionary contractions. Note, however, that both paths have positive slope, reflecting precautionary savings against the jump risk (both) and precautionary contractions risk (the case without diffusion hedging) .

Subplot (c) shows the the paths of the currents account deficits do not differ much in both scenarios. In both cases the current account deficit is "procyclical," in the sense that as the signal worsens the current account deficit shrinks. Of course, in our context income does not change with the signal but the point we are isolating is the sensitivity of consumption to news signifying a tightening of credit conditions. The analysis would not be changed in any substantive way by adding some correlation between such news and income shocks. We chose to keep income a deterministic process, in order to illustrate in the simplest possible way that variations in the price of exported commodities can have an effect above and beyond their direct income effect (which we have effectively "normalized" to 0 for simplicity), as long as they are useful in predicting the arrival of sudden stops.

Importantly, the procyclical behavior of the current account deficit does not reflect the same behavior of consumption in the two scenarios.

In the absence of diffusion risk hedging, it is consumption that does the adjustment. Chile is a point in case: When the price of copper collapsed at the end of the 1990s, Chile reduced rather than increased its current account deficit - exactly the opposite behavior of that of Australia, a developed economy which was facing similar terms of trade shocks. ${ }^{14}$ Moreover, the sharp decline in the current account deficit from 6 to about 3.5 percent of GDP toward the end of the sample, suggests that about half of the current account adjustment observed in Chile during the 1998-9 crisis can be accounted by optimal precautionary behavior in the absence of hedging. ${ }^{15}$

While the current account does follow a similar path when diffusive risk is hedged, the adjust-

\footnotetext{
${ }^{14}$ See Caballero, Cowan and Kearns (2005).

${ }^{15}$ The rest of the adjustment could be accounted for by excess adjustment (some have argued that the central bank contracted monetary policy excessively during this episode) and by the partial sudden stop itself (the "sovereign" spread tripled during this episode).
} 

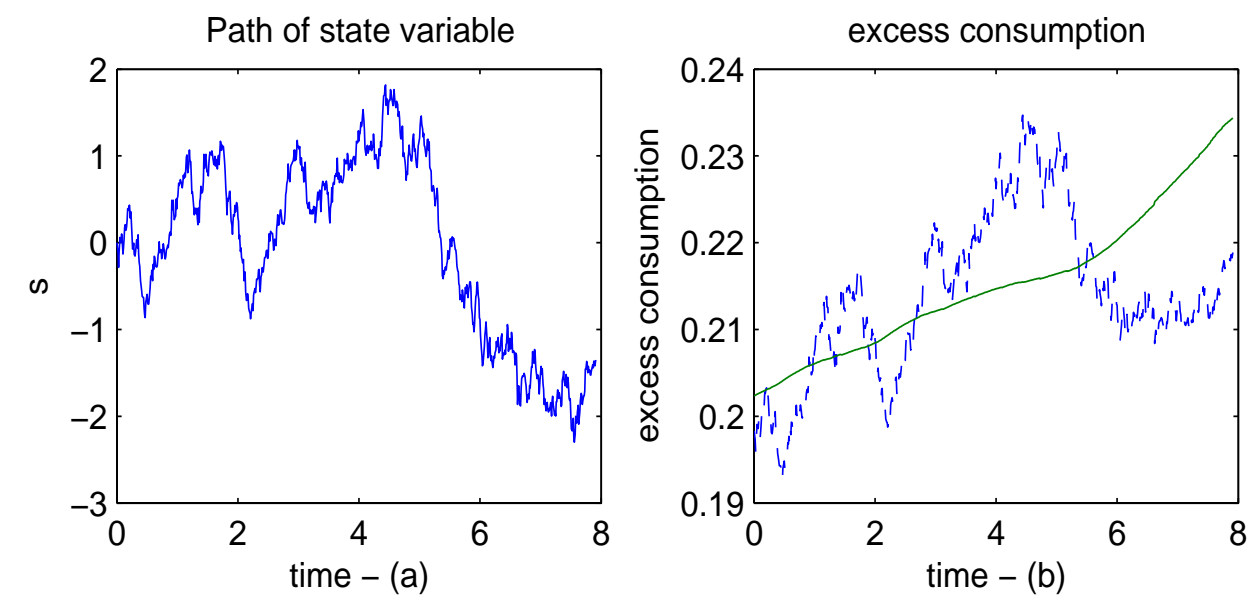

Path of current account/GDP
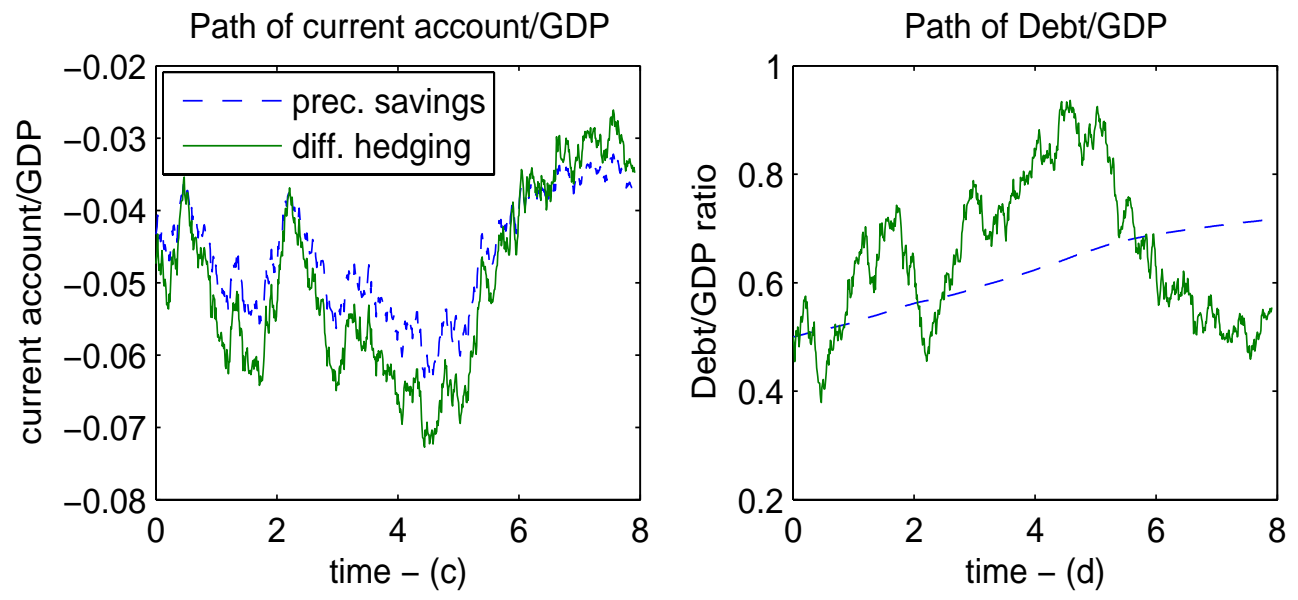

Figure 3: Subplot (a) depicts the path of the state variable (normalized by its yearly standard deviation) as a function of time. Subplot (b) depicts the path of excess consumption when income at time 0 is normalized to 1 and $\kappa=0.8$. The solid line depicts the path of excess consumption when the country uses diffusive hedging. The dashed line depicts the path of excess consumption when the country uses exclusively precautionary savings exclusively. Subplot (c) plots the current account $r X_{t}-c_{t}^{*}+y_{t}^{*}$ for the same two scenarios. Subplot (d) plots the path of the debt/gdp ratio for the two scenarios. Throughout the solid line refers to diffusive hedging and the dashed line to precautionary savings. 
ment variable is not consumption but the net asset position. To see this note that the current account is given by:

$$
C A_{t}=r X_{t}-c_{t}^{*}+y_{t}^{*}
$$

If this quantity remains practically unchanged across the two scenarios, it means that the behavior of $r X_{t}-c_{t}^{*}$ is roughly similar across them. However, as we have seen already, the volatility of $c^{*}$ is dramatically different across the two alternatives. This must mean that the net asset position $X_{t}$ has to become more volatile as we move from the precautionary savings case to diffusive hedging. Subplot (d) confirms this conjecture. In the absence of hedging, the country's net asset position $X_{t}$ is smooth, while it fluctuates when the country is hedging. As the state variable deteriorates, the debt to GDP ratio improves under hedging. Hence the increased volatility of the net asset position is just a natural consequence of what hedging is meant to achieve in the first place: Namely, to transfer resources between states of the world where they are less needed to states of the world where they are needed the most. As we increase hedging, a substantial portion of consumption volatility is "absorbed" by the procyclical variability of the net asset position.

Let us now turn to the second step and add the possibility of jump risk hedging. As we showed in Section 4, with full hedging possibilities excess consumption becomes constant prior to the sudden stop. This means that the country insulates itself from the sudden stop signal - it uses jump risk hedging to reduce the decline in consumption at the sudden stop, and diffusive hedging to offset fluctuations in the price of jump risk hedging as the signal changes. The top left subplot in Figure 4 reproduces the signal path in the previous figure, while the top right subplot illustrates the paths of the country's net foreign liabilities: The solid line shows the path of debt/gdp which fluctuates with the signal as the price of jump-risk insurance fluctuates. The important concept, however, is the dashed line which corresponds to the debt/gdp ratio the country will have if a sudden stop takes place. That it is, it includes the return from the jump-risk hedges. This dashed line is smooth and trending as the frictionless model would.

The bottom plots show the gains from jump risk hedging over and above diffusive hedging at the time of the sudden stop. To generate these panels we ran 500 simulations for ten years, and kept only those that yielded a sudden stop during that interval. The left panel shows the histogram of difference in consumption levels at the time of the sudden stop between the full hedging case and the diffusive-hedging only case. The right panel does the same for the consumption drops at 
the time of the sudden stop. Both panels have the common message that the possibility of jump risk hedging can significantly improve the outcome during a sudden stop.

\subsubsection{Welfare calculations}

We now summarize the different gains by evaluating the value functions of a country under different hedging scenarios. For this, let $V^{H}$ and $V^{H H}$ denote the value function of a country that has access to markets for diffusive risk only and to diffusive and jump risk, respectively. As a benchmark, $V$ denotes the value function of a country that only accumulates savings without accessing hedging markets of any kind. We now ask the question: How much resources would be required to compensate a country for its lack of access to hedging possibilities? Formally, when contrasting with the diffusive risk hedging only scenario, we solve for the amount $W$ such that:

$$
V^{H}\left(s_{t}, X_{t}, y_{t}^{*}\right)=V\left(s_{t}, X_{t}+W, y_{t}^{*}\right)
$$

We do the same for other scenarios as well (with $V^{H H}$ instead of $V^{H}$, and so on). It will be useful to define:

$$
w=\frac{W}{X_{t}+\frac{y^{*}}{r-g}}
$$

Then, using (17), (32), the definition of $w$ and (46) yields:

$$
d^{\gamma}=(1+w) b^{\gamma}
$$

where $b$ solves the differential equation (20) and $d$ is determined as in sections 4.4 or 4.3 depending on whether we want to allow jump risk hedging or not. Finally equation (48) leads to

$$
w=\left(\frac{d}{b}\right)^{\gamma}-1
$$

from which we can determine $W$ by using (47).

Figure 5 presents $W$ (normalized by gdp), when the country is allowed to hedge diffusive risks only, both diffusive and jump risks and when it is only allowed to accumulate precautionary savings. (Clearly in the third case the welfare gain is zero by construction) Finally we also report the welfare loss when the country ignores predictability altogether, as we described in section 3.3 and hence adopts suboptimal precautionary savings. ${ }^{16}$

\footnotetext{
${ }^{16}$ To determine the constant hazard $\bar{\lambda}$, we ran a simulation to determine the probability that the country enters
} 

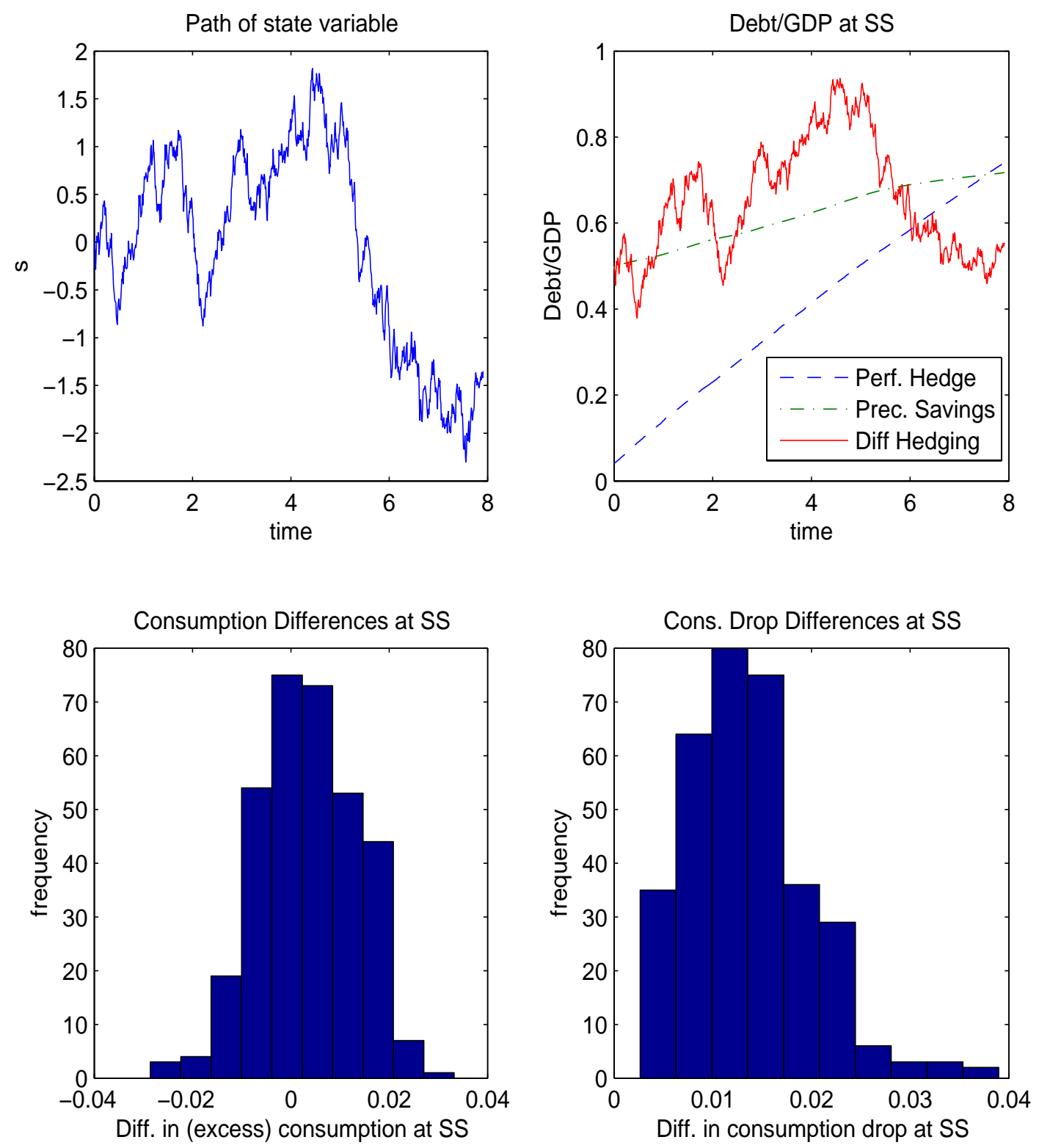

Figure 4: The top left subplot is identical to the top left plot of figure 3. It depicts the path of the signal normalized by its yearly standard deviation. The top right subplot depicts debt to GDP as a function of time. The various lines capture the level of debt to GDP assuming the country were to go into a sudden stop at time $t$. The bottom left figure depicts results from a simulation. Conditional on $s_{0}=0$, we draw 500 paths for a duration of 10 years. Keeping those paths where the country enters in to a SS within 10 years the bottom left subplot shows the differences in the level of consumption at the time of the sudden stop between the perfect hedging scenario and the diffusive hedging scenario. Similarly, the bottom right figure shows the differences in consumption drop upon entering the sudden stop between the two scenarios. 
The figure shows that for a range of values of $s$ corresponding to the range of the (log) price of copper in our sample, the gains from hedging are substantial. Using hedging in place of precautionary savings typically results in values of $W$ between 8 and $10 \%$ of GDP, if only "diffusive" hedging is used and are potentially up to $18 \%$ when both "jump" and "diffusive" hedging is used. To put this in perspective, notice that the debt/gdp ratio for the country under consideration is $50 \%$. This implies that using hedging instead of precautionary savings is equivalent to reducing the debt to GDP ratio of the country from $50 \%$ to a number between $32 \%$ and $40 \%$.

Another important observation about the figure is that most of the benefits of hedging accrue for intermediate values of the state variable. As the state variable approaches $\pm \infty$ all hedging strategies start becoming irrelevant. The reason is intuitive. If $s_{t} \rightarrow \infty$ then the hazard rate goes to 0 and the expected time to the next sudden stop approaches infinity. Hence, the sudden stop is "very far away". Therefore any comparative benefits of one strategy over the other get so heavily discounted, that they become essentially 0 . By a similar reasoning, as $s_{t} \rightarrow-\infty$ the sudden stop becomes imminent (and insurance against it too expensive).

A reassuring feature of Figure 5 is that the welfare benefits of only hedging diffusive risks is significant and covers more than half of the distance to perfect hedging, irrespective of the level of the state variable. This suggests that the benefits of hedging will accrue even when there is no explicit insurance against jump risk. This is important since developing markets for jump (sudden stop) risk is substantially harder than for diffusive risk, which are readily available in many cases.

Finally, note from the lowest line (with crosses) that ignoring hedging and the variability of the sudden stop hazard — essentially accumulating precautionary savings at a constant rate - is not too costly when the likelihood of a sudden stop is high as long as the country has the right sense of the unconditional (on the signal) likelihood of a crisis: neither precautionary savings only strategy is good at handling the sudden stop. However the cost of a non-contingent precautionary savings strategy takes places during good times (high $s$ ), when the country overaccumulates costly non-contingent resources when the low risk of a sudden stop does not justify it.

into a sudden stop in the next 10 years conditional on $s_{0}=0$. We then determined the constant hazard rate $\bar{\lambda}$ so that it will produce exactly the same probability. 


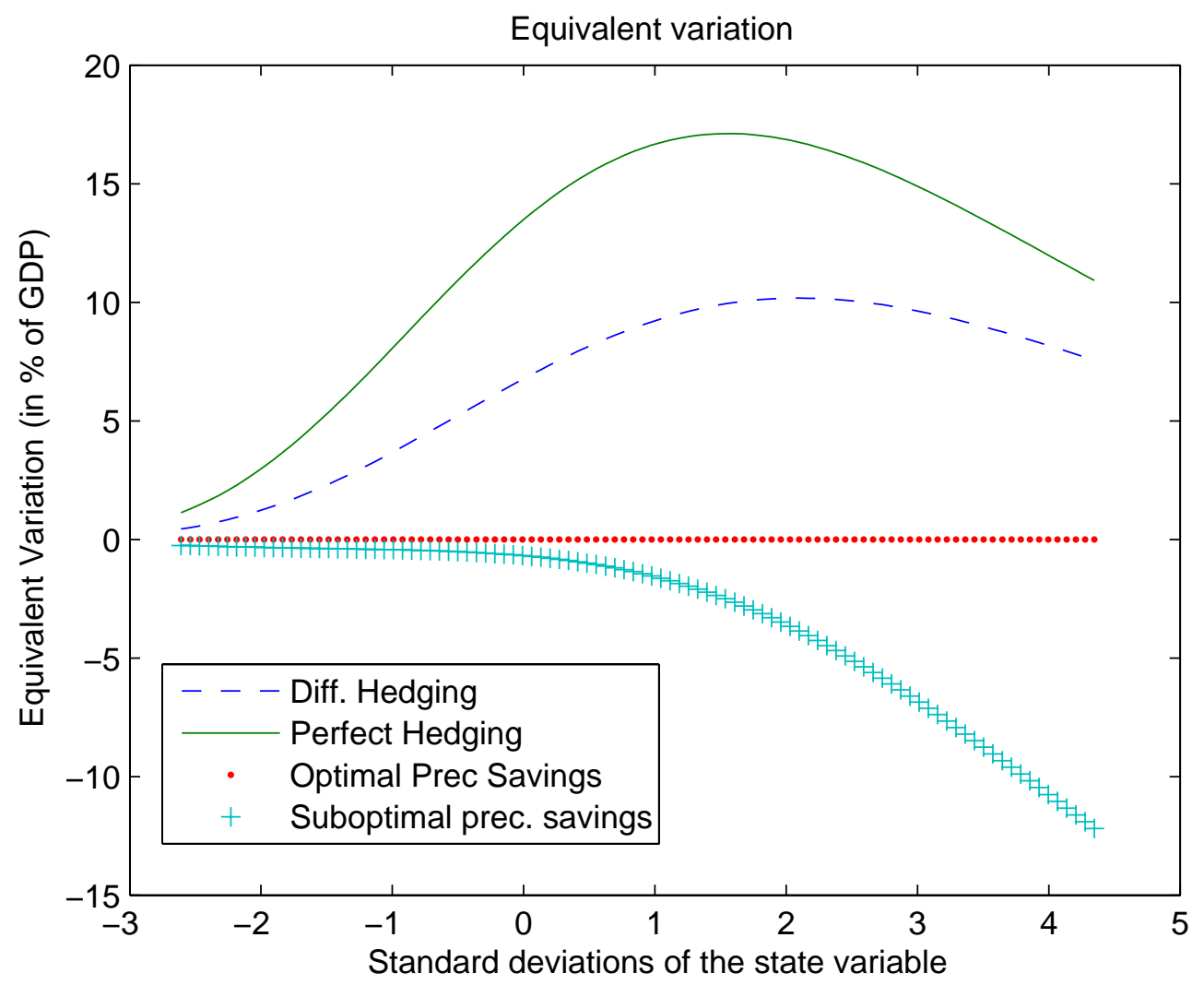

Figure 5: Equivalent variation for a "typical" range of values of $s_{t}$ (normalized by its yearly standard deviation). For a precise definition of the notion of equivalent variation see the text. 


\subsubsection{Decomposing the Gains}

Let us not decompose the gains from different hedging strategies. Our model produces a clear demarcation of time into three stages: Before a sudden stop, during a sudden stop, and after a sudden stop. The marginal value of a dollar is highest during the sudden stop, somewhat lower in the phase that precedes it and lowest in the post-sudden stop phase. Hence, a strategy produces welfare gains if it manages to "shift" resources into the sudden stop and also into the phase before the sudden stop.

Table 2 reports the results of a simple simulation exercise: We start with $s_{0}=0$, and simulate a number of paths for $s_{t}$ for 10 years. We also simulate the arrival of sudden stops by drawing independent exponential variables and using formula (11) to determine the time of arrival of sudden stops. In about $67 \%$ of the simulated paths for $s_{t}$ we observe a sudden stop within 10 years. We then compute the behavior of $c_{t}^{*}, X_{t}$ along the path, consumption immediately following the sudden $\operatorname{stop} c_{\tau+}^{*}$ and the associated consumption drop upon entering the sudden stop. Results are reported for the four strategies of the previous section from worst to best: suboptimal precautionary savings, optimal precautionary savings, diffusive hedging only, and perfect hedging. The first column shows initial (excess) consumption, which is highest for perfect hedging and second highest for diffusive hedging. The reason for this is that perfect hedging does not require precautionary savings, while that of diffusive hedging requires less precautionary savings than the other two. It is the next three columns that capture the most important results, however. The second column shows the level of consumption at the time of the sudden stop, with a clear advantage for the hedged strategies; the third column shows that the drop of consumption at the time of the sudden stop is significantly lower in the case of diffusive and jump risk hedging. The reason why the difference in drops is greater than that of levels at the time of sudden stop is important: In all but the full insurance case, consumption rises over time as precautionary savings accumulate. But this also means that in all the cases but the jump risk hedge case, the economy is relatively vulnerable if the sudden stop happens in the near future, when the country has not had the time to accumulate resources. This is reflected in the last column of the table, which shows the correlation between the time it takes for the sudden stop to arrive and the level of consumption at the sudden stop. With jump risk hedge, there is no correlation. With all the rest, this correlation is positive, although diffusive hedging can also reduce it significantly. 


\begin{tabular}{lcccc}
\hline \hline & $c^{*}(0)$ & mean $c^{*}(\tau+)$ & mean $\Delta c_{\tau}^{*}$ & $\operatorname{cov}\left(c_{\tau}^{*+}, \tau\right)$ \\
\hline Suboptimal Precautionary Savings & 19.99 & 12.00 & 9.39 & 0.99 \\
Optimal Precautionary Savings & 19.83 & 12.04 & 8.37 & 0.98 \\
Only Diffusive hedging & 20.24 & 12.66 & 9.00 & 0.67 \\
Diffusive+Jump hedging & 20.64 & 13.02 & 7.60 & 0 \\
\hline \hline
\end{tabular}

Table 2: Initial (excess) consumption $c^{*}(0)$, (excess) consumption at the time of the sudden stop $c_{\tau^{+}}^{*}$, standard deviation of the consumption path leading to the sudden stop $\left(\operatorname{std}\left(c_{t}^{*}\right)\right)$ and instantaneous consumption drop upon entering the sudden stop $\left(\Delta c_{\tau}^{*}\right)$ for various hedging scenarios.

\subsubsection{Introducing risk premia}

Up to now we have assumed no risk-premia on either diffusive or jump risk hedging. In this section we go to the opposite extreme and ask: how large would the risk premia have to be for the country to give up hedging?

The framework we developed in Section 6 is well suited to answer this question. In particular, equation (45) shows that the "critical" risk premium that would render diffusive hedging pointless is:

$$
\phi_{1}=-\gamma \sigma^{2} \frac{d_{s}}{d}
$$

and equation (44) shows that the critical overestimation of the hazard (risk premium) for "jump" hedging is:

$$
\phi_{2}=\frac{K}{(r d)^{\gamma}}-1
$$

Since $d=b$ if $p=0$ (no diffusive risk hedging) and $I=0$ (no jump risk hedging) we can replace $d$ with the function $b$ from Section 3.

Figure 6 reports the results when one computes the "critical risk premiums" $\phi_{1}$ and $\phi_{2}$ as implied by the above formula, for the range of $(\log )$ price of copper observed in our sample. The top panel shows that the critical risk premium for diffusive hedging is about $8 \%$. The bottom panel shows that the critical risk premium for jump risk hedging typically exceeds $100 \%$. These large critical premia confirm the benefits of hedging. For diffusive risks, which in many cases already have up-and-running markets, $8 \%$ is very large when compared with reasonable premia in liquid 

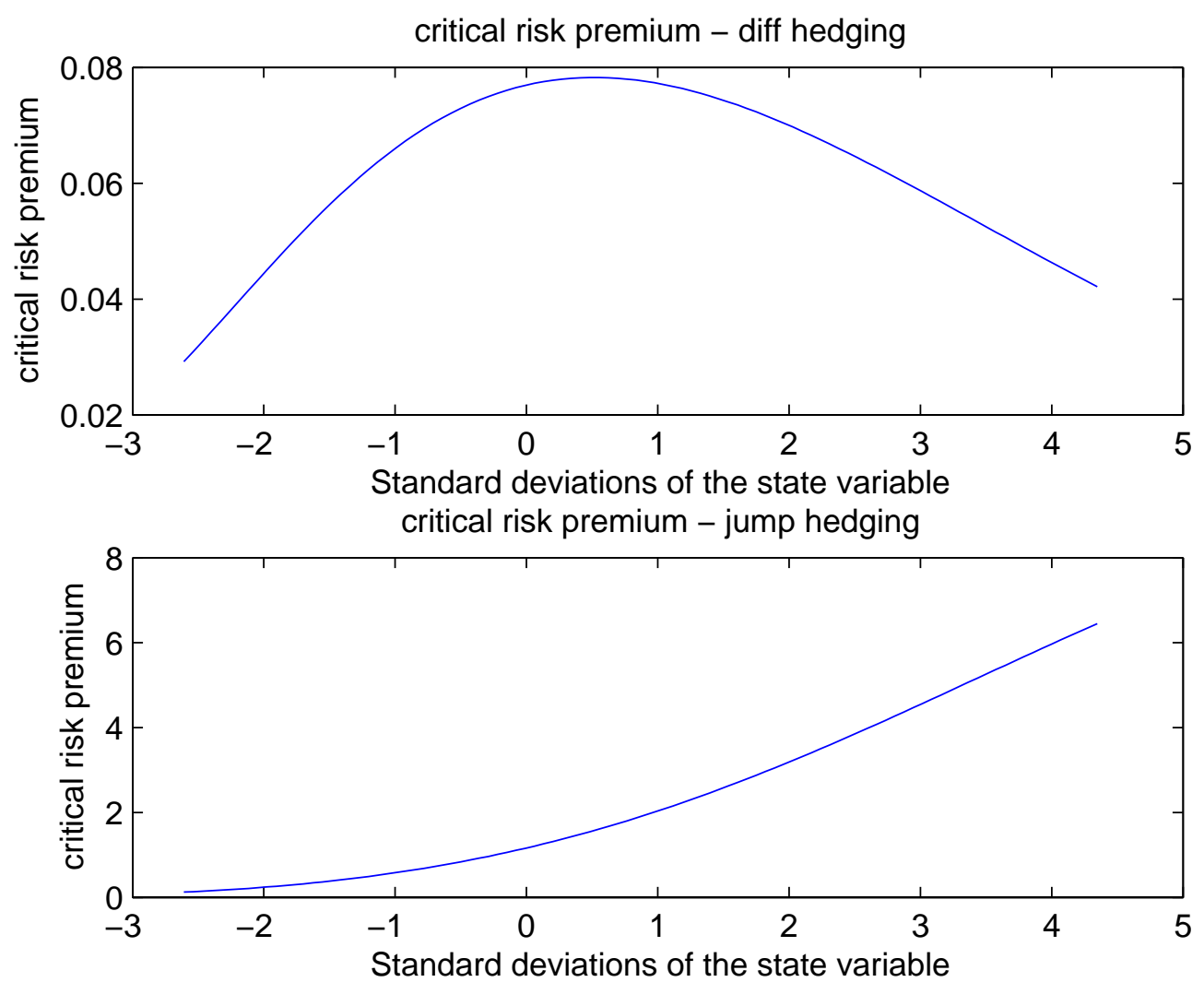

Figure 6: Critical Risk Premia for diffusive hedging and jump hedging. The top plot depicts the critical risk premium $\phi_{1}=-\gamma \sigma^{2} \frac{d_{s}}{d}$ which would make it optimal to avoid diffusive hedging altogether. The bottom line depicts $\phi_{2}=\frac{K}{(r d)^{\gamma}}-1$, i.e. the percentage by which the true hazard rate would have to be "over-estimated" by insurers in order to make jump hedging too expensive. 
markets. ${ }^{17}$ For jump risk hedging, a premium above $100 \%$ reflects the large potential gains of developing such markets.

\section{Sudden stops: Time variation in hazard rates and traded vari- ables}

In this section we argue that Chile is not an exception in terms of the usefulness of our analysis. For this, we document two important ingredients for our framework: that there is a significant element of predictability in sudden stops, and that this predictability is correlated with readily available and tradable indices which are largely beyond the control of individual emerging market economies (hence moral hazard is of limited concern). Moreover, we conduct this analysis in a simple reduced form framework, so that our conclusions here are not intertwined with our specific methodology in the rest of the paper.

\subsection{Time Variation and Predictability of the Likelihood of Sudden Stops}

We identify the sudden stop hazard using the data and methodology from Calvo, Izquierdo and Mejia (2004). They identify sudden stops based on a number of cutoffs and indicators, which they then use to run (probit) regressions against country specific variables (dollarization, openness etc.) and time effects. Their data are yearly and span the period 1990-2001 for 32 countries, of which 15 are emerging markets (Argentina, Brazil, Chile, Colombia, Czech Republic, Ecuador, Indonesia, Mexico, Nigeria, Peru, Philippines, South Africa, South Korea, Thailand and Turkey). We focus exclusively on the 15 emerging markets.

We essentially replicate the Calvo et al steps by running logit and probit regressions of the form:

$$
y_{i t}=\operatorname{Pr}(S S=1)=F\left(x_{i t}^{\prime} \beta\right)
$$

We use the most significant variables reported in their paper, namely the degree of dollarization, openness, and terms of trade, as well as time fixed effects. After running the regressions, we isolate the predicted probabilities $F\left(x^{\prime} \widehat{\beta}\right)$ and the actual regressor values $x^{\prime} \widehat{\beta}$.

\footnotetext{
${ }^{17}$ For example, the corresponding Sharpe ratio is 0.35 which is nearly twice the Sharpe ratio for the long sample of the US stock market (see, e.g. Campbell and Cochrane (1999)).
} 
Assuming that the conditional probabilities $F\left(x^{\prime} \widehat{\beta}\right)$ capture well the variation in the likelihood of running into a sudden stop, we investigate whether there is significant time variation in the hazard rates. Columns 1 and 2 of Table 3 show that indeed the bulk of the variation is in the within (across time) dimension as opposed to the between (across country) variation. Also, the standard deviation of 0.24 indicates that the conditional probabilities of sudden stops vary substantially across time.

Time variability in the conditional probabilities is however not synonymous with predictability. If there is little or no serial correlation in the conditional probabilities, then there is little benefit from time varying precautionary accumulation of reserves, hedging etc. All these measures are effective if there is some persistence in the process that generates the conditional probabilities. The next four rows in Table 3 report results from a dynamic panel data regression of the form:

$$
y_{i t}=\alpha_{i}+\beta_{1} y_{i t-1}+\beta_{2} y_{i t-2}+\varepsilon_{i t}
$$

Rows 2 and 3 are simple fixed effects regressions with robust standard errors. The next two rows report the equivalent results when one uses the estimator of Arellano and Bond (1999), to avoid the well known biases of dynamic panel data models. Uniformly, the results indicate substantial persistence and hence predictability of the conditional probabilities.

\subsection{Traded Variables and the variation of the conditional likelihood of Sudden Stops}

Sofar we have only examined whether there appears to be enough variation and persistence in the conditional probability of sudden stops to justify time varying precautionary measures. A separate question is whether traded instruments can be used to hedge away these risks. One way to answer this question is to investigate whether there exist traded instruments that can "predict" the time variation in the conditional probabilities. Table 4 addresses this question.

We use $H Y_{t-1}$ to denote the difference between Aaa and Baa bonds (high yield spread) as reported in the historical series H.15 of the Federal Reserve. Columns (1) and (2) use the predicted

values $x^{\prime} \widehat{\beta}$ and $F\left(x^{\prime} \widehat{\beta}\right)$ obtained using a probit model similar to Calvo et al. (2004) to run the regressions: 


\begin{tabular}{|c|c|c|c|c|}
\hline & Logit & Probit & Logit & Probit \\
\hline & $(1)$ & $(2)$ & $(3)$ & $(4)$ \\
\hline Between /Within & $0.06 / 0.24$ & $0.07 / 0.23$ & & \\
\hline \multirow[t]{2}{*}{ Prob (Lag1) } & & & 0.51 & 0.53 \\
\hline & & & $(5.32)^{* *}$ & $(5.45)^{* *}$ \\
\hline \multirow[t]{2}{*}{ Prob (Lag2) } & & & -0.48 & -0.48 \\
\hline & & & $(-5.89)^{* *}$ & $(-5.89)^{* *}$ \\
\hline \multirow[t]{2}{*}{ Prob (Lag1) Arellano -Bond } & & & 0.46 & 0.46 \\
\hline & & & $(4.64)^{* *}$ & $(4.65)^{* *}$ \\
\hline \multirow[t]{2}{*}{ Prob (Lag2) Arellano - Bond } & & & -0.67 & -0.68 \\
\hline & & & $(-5.12)^{* *}$ & $(-5.30)^{* *}$ \\
\hline R-squared & & & 0.37 & 0.37 \\
\hline
\end{tabular}

Table 3: Columns (1) and (2): Ratio of variation (reported as ratio of standard deviations) attributable to the within (accross time) dimension as opposed to the between (accross panel) dimension. Columns (3) and (4): Regressions of predicted probabilities on their lagged values using regular regession and the Arellano-Bond estimator. 


\begin{tabular}{lcccc}
\hline \hline & $(1)$ & $(2)$ & $(3)$ & $(4)$ \\
\hline \multirow{2}{*}{ L.HY } & D.regressors & D.lprob & D.regressors & D.lprob \\
& -16.90 & -83.30 & -71.62 & -70.20 \\
& $(-4.54)^{* *}$ & $(-4.30)^{* *}$ & $(-4.53)^{* *}$ & $(-4.40)^{* *}$ \\
\hline Observations & 135 & 135 & 135 & 135 \\
R-squared & 0.20 & 0.17 & 0.19 & 0.19 \\
\hline \hline
\end{tabular}

Table 4: Column (1): Regressions of first differences in the regressors $x_{i t}^{\prime} \beta$ used in Calvo et al. (2004) on the lagged difference between Baa and Aaa bonds (L.HY). Column (2) Same as column (1) with the sole exception that we use differences in the (log) predicted probability $\log . F\left(x_{i t}^{\prime} \beta\right)$ in place of $x_{i t}^{\prime} \beta$. Columns (3)-(4): Same as columns (1)-(2) using a logit instead of a probit model. For all regressions we use panel corrected standard errors allowing for heteroskedasticity and contemporaneous correlation across panels. A constant is included but not reported.

$$
\begin{aligned}
\Delta\left(x_{i t}^{\prime} \widehat{\beta}\right) & =\alpha_{0}+\alpha_{1} H Y_{t-1}+\varepsilon_{i t} \\
\Delta \log \left(F\left(x_{i t}^{\prime} \widehat{\beta}\right)\right) & =\alpha_{0}+\alpha_{1} H Y_{t-1}+\varepsilon_{i t}
\end{aligned}
$$

The first regression is a projection of the change in the regressors entering the probit model of Calvo et. al. (2004) on the high yield spread at $t-1$. The second regression performs a similar exercise for the predicted probabilities. If, for instance, increases in the high yield spread coincide with periods of high risk and risk aversion, then emerging markets might be exposed to contagion risk. Hence, in a manner similar to copper for Chile, variations in the $H Y$ can be used to form a hedging strategy.

As Table 4 shows, the R-squared of these regressions is close to $20 \%$. Hence, $20 \%$ of the innovations in the conditional probabilities are predicted by a variable that belongs to the information set of agents at time $t-1$ and has a liquid market. To put this in perspective, in Table 3 the total predictable variation in the conditional probabilities was about $37 \%$ of their variation in the sample. Comparing the two numbers reveals a substantial co-variation between the predicted hazard and $H Y_{t-1}$.

Note that the coefficient has the expected sign since $H Y$ is mean reverting, thus a high degree 
of $H Y$ today predicts a lower $H Y$ tomorrow and hence a lower likelihood of a sudden stop. To see this point more clearly, suppose that there is positive contemporaneous correlation between $\Delta x_{i t}^{\prime} \widehat{\beta}$ and $\Delta H Y_{t}$, i.e. suppose that:

$$
E\left(\Delta x_{i t}^{\prime} \widehat{\beta}\right)=\alpha_{0}+\widetilde{\alpha}_{1} E\left(\Delta H Y_{t}\right) \text { with } \widetilde{\alpha}_{1}>0
$$

Now assume that $H Y_{t}$ is a simple $\mathrm{AR}(1)$ process:

$$
H Y_{t}=\rho H Y_{t-1}+\varepsilon_{V, t} \quad \text { with } \rho<1
$$

so that:

$$
\Delta H Y_{t}=H Y_{t}-H Y_{t-1}=(\rho-1) H Y_{t-1}+\varepsilon_{V, t}
$$

and also:

$$
E\left(\Delta H Y_{t}\right)=(\rho-1) H Y_{t-1}
$$

Plugging (50) into (49) shows that:

$$
E\left(\Delta x_{i t}^{\prime} \widehat{\beta}\right)=\alpha_{0}+\widetilde{\alpha}_{1}(\rho-1) H Y_{t-1}
$$

Hence the regressions in Table 4 produce a negative sign on lagged $H Y$ since $\widetilde{\alpha}_{1}(\rho-1)<0$.

The main reason why $H Y$ performs well in terms of predictive power is that it captures well the common variation of the arrival of sudden stops across countries. To illustrate this, we computed the average yearly difference in the probability of a sudden stop as implied by the probit model. Then we plotted the (negative of) the high yield (lagged once) against it. Figure 7 illustrates the strong comovement between the two series.

\section{$7 \quad$ Final Remarks}

In this paper we characterized several aspects of sudden stops, precautionary contractions, and the corresponding aggregate hedging strategies. For this, we built a model simple enough to shed analytical light on some of the key issues but realistic enough to provide some quantitative guidance.

We showed that even after removing all other sources of uncertainty, the optimal path of aggregate demand in an unhedged emerging market economy exhibits large precautionary contractions. That is contractions that precede sudden stops. We then argued that smooth diffusive hedging could remove much of these fluctuations even in the absence of more complex jump hedging mechanisms. 


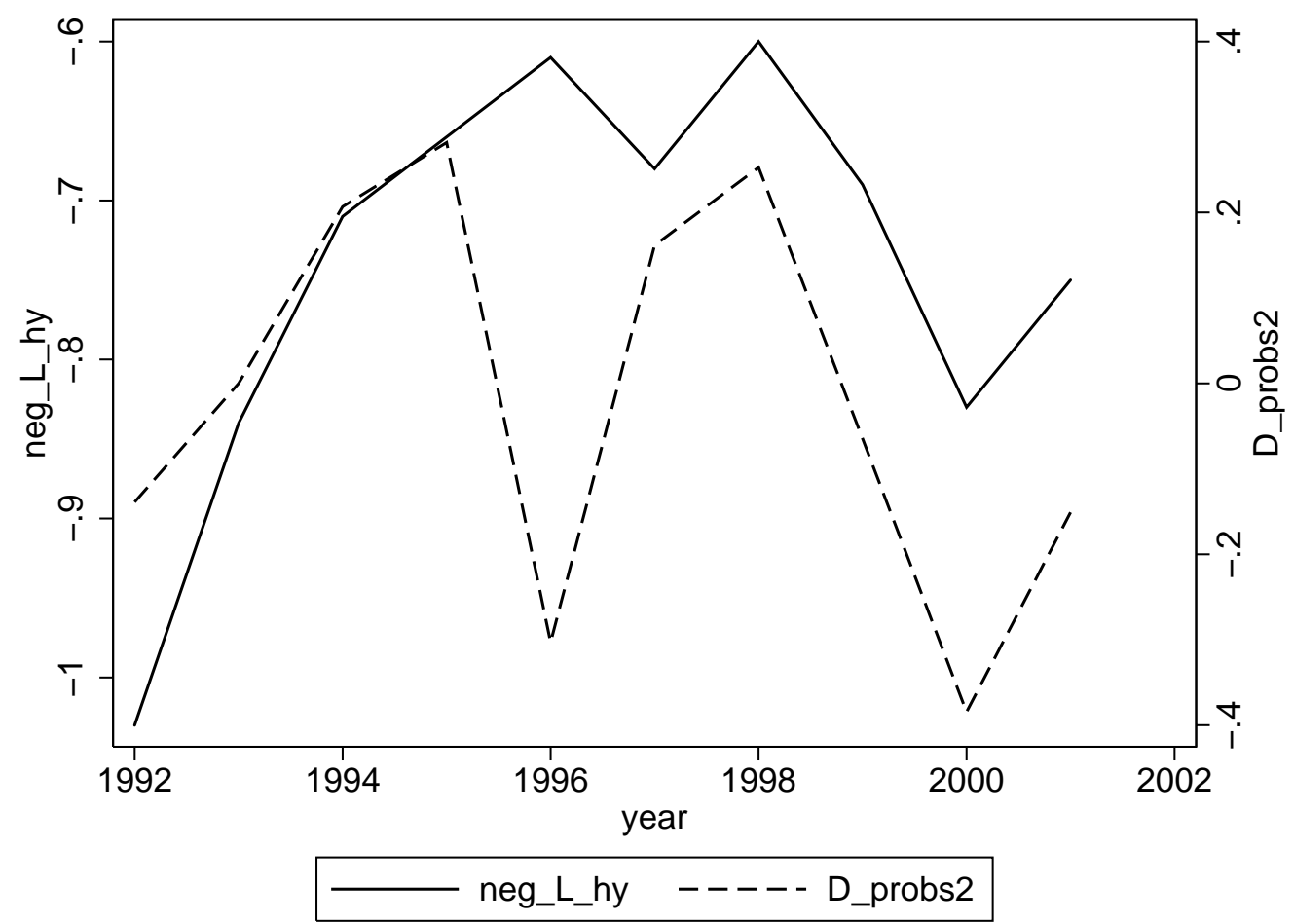

Figure 7: The joint variation in the average yearly difference in the probability of a sudden stop ( $D \_$probs) as implied by a probit model and the (negative of) the high yield spread (lagged once) $($ neg_l_hy). 
The gains from these hedging strategies are large, which raises the question of why aren't countries already doing this in a significant manner. Our somewhat informed belief is that the problem is mostly one of coordination, which could be reduced significantly with the involvement of the international financial institutions (IFIs).

On one end, hedging along the lines we have suggested is not standard practice for external liability management: Any sensible policymaker knows that the political (and personal) cost of something going wrong while implementing an unorthodox strategy is many times larger than that of failing while following the standard recipe. The IFIs can help by modernizing their advice on standard practices.

On the other, while one can imagine many indicators that could be used to start improving hedging strategies immediately, such as the price of commodities, the high yield spread (HY), the volatility index (VIX), indicators of US and other developed regions activity, and so on, some of these markets are not nearly as large as they would need to be (recall that the issue is not to hedge the income effect of commodity price fluctuations but the sudden stop, which is significantly lager, and probably not just as a multiplier but also as a bellwether). Market creation requires coordination, and the development of a few general indices that can ensure adequate liquidity. Again, the IFIs can help develop these markets. For example, the IMF could modify its contingent credit lines to index them to indicators that could eventually be adopted by the private sector and traded in open markets. 


\section{References}

Albert, J. H., And S. Chib (1993): "Bayes Inference via Gibbs Sampling of Autoregressive Time Series subject to Markov mean and Variance Shifts," J. Bus. Econ. Stat., 11(1), 1-15.

Burnside, C., M. Eichenbaum, and S. Rebelo (2003): "Prospective Deficits and the South East Asian Crisis," Journal of Political Economy - forthcoming.

Caballero, R. J. (2001): Macroeconomic Volatility in Reformed Latin America., vol. 25. InterAmerican Development Bank, Washington, D.C., first edn.

Caballero, R. J., K. Cowan, and J. Kearns (2005): "Fear of Sudden Stops: Lessons from Australia and Chile," mimeo. MIT.

Caballero, R. J., and A. Krishnamurthy (2001): "International and Domestic Collateral Constraints in a Model of Emerging Market Crises," Journal of Monetary Economics., 48(3), $513-548$.

(2003): "Excessive Dollar Debt: Financial Development and Underinsurance," Journal of Finance.

Caballero, R. J., and S. Panageas (2003): "Hedging Sudden Stops and Precautionary Recessions: A quantitative framework," NBER Working Paper.

(2005): "A Quantitative Model of Sudden Stops and External Liquidity Management," NBER Working Paper w11293.

Calvo, G., A. Izquierdo, and L.-F. Mejía (2004): "On the empirics of Sudden Stops: the relevance of balance-sheet effects," Mimeo, IADB.

Cochrane, J., and J. Campbell (1999): "By Force of Habit: A Consumption-Based Explanation of Aggregate Stock Market Behavior," Journal of Political Economy, 107, 205-251.

Cox, J. C., And C.-F. Huang (1989): "Optimal consumption and portfolio policies when asset prices follow a diffusion process," J. Econom. Theory, 49(1), 33-83. 
Diebold, F. X., J.-H. Lee, and G. Weinbach (1994): Regime Switching with Time-Varying Transition Probabilities in Nonstationary Time Series Analysis and Cointegrationchap. 10, pp. 283-302. Oxford University Press.

Duffie, D., L. Pedersen, and K. Singleton (2000): "Modeling Sovereign Yield Spreads: A Case Study of Russian Debt," Graduate School of Business, Stanford University, forthcoming, Journal of Finance.

Fleming, W. H., And H. M. Soner (1993): Controlled Markov processes and viscosity solutions, vol. 25 of Applications of Mathematics. Springer-Verlag, New York, first edn.

Froot, K. A., D. S. Scharfstein, and J. Stein (1989): "LDC Debt: Forgiveness, Indexation, and Investment Incentives," Journal of Finance, 44(5), 1335-50.

Haldane, A. (1999): "Private Sector Involvement in Financial Crisis: Analytics and Public Policy Approaches," Financial Stability Review, 7.

Hamilton, J. D. (1989): "A new approach to the economic analysis of nonstationary time series and the business cycle," Econometrica, 57(2), 357-384.

Harrison, J. M. (1985): Brownian motion and stochastic flow systems, Wiley Series in Probability and Mathematical Statistics: Probability and Mathematical Statistics. John Wiley \& Sons Inc., New York.

Karatzas, I., J. P. Lehoczky, and S. E. Shreve (1987): "Optimal portfolio and consumption decisions for a "small investor" on a finite horizon," SIAM J. Control Optim., 25(6), 1557-1586.

Karatzas, I., And S. E. Shreve (1991): Brownian motion and stochastic calculus, vol. 113 of Graduate Texts in Mathematics. Springer-Verlag, New York, second edn.

Karatzas, I., And H. Wang (2000): "Utility maximization with discretionary stopping," SIAM J. Control Optim., 39(1), 306-329.

Kim, C. J., and C. R. Nelson (1998): "Business cycle turning points, a new coincident index, and tests of duration dependence based on a dynamic factor model with regime switching," Rev. Econ. Statist., 80(1), 188-201. 
Kim, C.-J., And C. R. Nelson (1999): State-space models with regime switching Classical and Gibbs-sampling approaches with applications. MIT Press, Cambridge MA and London.

Krugman, P. (1988): "Financing vs. Forgiving a Debt Overhang," Journal of Development Economics, 29, 253-68.

Robert, C. P., and G. Casella (1999): Monte Carlo statistical methods. Springer-Verlag, New York.

Tirole, J. (2002): "Inefficient Foreign Borrowing: A Dual-and-Common Agency Perspective," Mimeo, Toulouse.

Verhulst, F. (1996): Nonlinear differential equations and dynamical systems, Universitext. Springer-Verlag, Berlin, second edn. 


\section{A Appendix}

\section{A.1 Proofs for section 2.2}

Proof. (Lemma 1) Since we made the simplifying assumption that the country suffers only one financial crisis, the maximization problem post sudden stop $(t>\tau+T)$ is simply:

$$
\begin{aligned}
V^{+}\left(X_{\tau+T}, y_{\tau+T}^{*}\right)= & \max _{c_{t}^{*}} \int_{\tau+T}^{\infty} \frac{c_{t}^{* 1-\gamma}}{1-\gamma} e^{-r(t-(\tau+T))} d t \\
& \text { s.t. } \\
& \int_{\tau+T}^{\infty} c_{t}^{*} e^{-r(t-(\tau+T))} d t=X_{\tau+T}+\int_{\tau+T}^{\infty} y_{t}^{*} e^{-r(t-(\tau+T))} d t
\end{aligned}
$$

This problem has the trivial solution:

$$
c_{t}^{*}=r W_{2}^{e} \quad \tau+T<t<\infty
$$

with

$$
W_{2}^{e}=X_{\tau+T}+\frac{y_{\tau+T}^{*}}{r-g} .
$$

This constant can be interpreted as the "excess" wealth at date $\tau+T$ (that is, the wealth in excess of that which is needed to cover the reservation-consumption level $\kappa y_{t}$ ). During the sudden stop $(\tau \leq t \leq \tau+T)$ we can use the continuation value function, $V\left(X_{\tau+T}, y_{\tau+T}^{*}\right)$, from above, to write the maximization problem for the sudden stop phase:

$$
\begin{aligned}
V^{S S}\left(X_{\tau}, y_{\tau}^{*}\right)= & \max _{c_{t}^{*}} \int_{\tau}^{\tau+T} \frac{c_{t}^{* 1-\gamma}}{1-\gamma} e^{-r(t-\tau)} d t+e^{-r T} V^{+}\left(X_{\tau+T}, y_{\tau+T}^{*}\right) \\
& \text { s.t. } \\
& \int_{\tau}^{\tau+T} c_{t}^{*} e^{-r(t-\tau)} d t=X_{\tau}+\int_{\tau}^{\tau+T} y_{t}^{*} e^{-r(t-\tau)} d t-e^{-r T} X_{\tau+T} \\
& X_{\tau+T} \geq \bar{X}_{\tau+T} .
\end{aligned}
$$

where

$$
\bar{X}_{\tau+T}=\zeta\left(X_{\tau}+\frac{y_{\tau}^{*}\left(1-\frac{e^{g T}}{\zeta}\right)}{r-g}\right)
$$

by (5). Since the country is growing and wishes to expand its consumption at a faster rate than the constraint allows, it can be easily shown ${ }^{18}$ that the sudden stop constraint, (55), is always binding

$$
X_{\tau+T}=\bar{X}_{\tau+T}
$$

\footnotetext{
${ }^{18}$ For details see Caballero and Panageas (2003)
} 
Given this result, the optimization problem is straightforward. It is a deterministic consumption problem subject to a final wealth condition. A few steps of algebra show that:

$$
c_{t}^{*}=\frac{r}{1-e^{-r T}} W_{1}^{e}, \quad \tau<t<\tau+T
$$

with

$$
W_{1}^{e}=X_{\tau}+\frac{y_{\tau}^{*}\left(1-e^{-(r-g) T}\right)}{r-g}-e^{-r T} \bar{X}_{\tau+T} .
$$

It is easy to see from these expressions that as $\bar{X}_{\tau+T}$ rises, $c_{t}^{*}$ falls. The country has to cut back consumption during the crisis in order to satisfy a tighter sudden-stop constraint. We are now ready to determine the sudden-stop value function:

$$
\begin{aligned}
V^{S S}\left(X_{\tau}, y_{\tau}^{*}\right) & =\int_{\tau}^{\tau+T} \frac{\left(r W_{1}^{e}\right)^{1-\gamma}}{1-\gamma} e^{-r(t-\tau)} d t+e^{-r T} \int_{\tau+T}^{\infty} \frac{\left(r W_{2}^{e}\right)^{1-\gamma}}{1-\gamma} e^{-r(t-(\tau+T))} d t \\
& =\frac{\left(r W_{1}^{e}\right)^{1-\gamma}}{1-\gamma}\left(\frac{1-e^{-r T}}{r}\right)+\frac{\left(r W_{2}^{e}\right)^{1-\gamma}}{1-\gamma}\left(\frac{e^{-r T}}{r}\right) .
\end{aligned}
$$

It is easy to verify that our careful choice of the constraint pays off at this stage. The value function simplifies to:

$$
\begin{aligned}
V^{S S}\left(X_{\tau}, y_{\tau}^{*}\right) & =K\left(\frac{1}{r}\right)^{\gamma} \frac{\left(X_{\tau}+\frac{y_{\tau}^{*}}{r-g}\right)^{1-\gamma}}{1-\gamma} \\
& =K V^{+}\left(X_{\tau}, y_{\tau}^{*}\right)
\end{aligned}
$$

where $V\left(X_{\tau}, y_{\tau}^{*}\right)$ denotes the value function in the absence of a sudden-stop constraint and $K$ is the constant given in the statement of the proposition. $K>1$ is a straightforward consequence of the fact that the value function (which is negative if $\gamma>1$ ) has to be lower than the value function in the absence of the constraint (55) A simple differentiation shows that $K_{\zeta}>0$ as long as $\gamma>1$.

\section{A.2 Proofs for section 3.1}

Proof. (Lemma 2) We give a brief sketch of the proof. Differentiate the left hand side of (18) with respect to $b$ to obtain:

$$
-r+(1-\gamma) \frac{\lambda}{\gamma}\left(\frac{\underline{b}}{b}\right)^{\gamma}<0
$$


since $\gamma>1$. Moreover, at $b=\frac{1}{r}$ the left hand side of (18) is greater than 0 while at $b=\underline{b}$ it is less than 0 . This establishes existence and uniqueness of the solution to (18) by the intermediate value Theorem.

\section{A.3 Propositions and Proofs for section 3.2}

Proposition 5 If there exists a function b(s) solving (20) subject to (21) and (22) then (17) solves (15) and is the value function of the problem as long as:

$$
\lim _{t \rightarrow \infty} E\left[e^{-r t} 1\{\tau>t\} b(s)^{\gamma} \frac{\left(X_{t}^{o p t}+\frac{y_{t}^{*}}{r-g}\right)^{1-\gamma}}{1-\gamma}\right]=0
$$

Proof. Substituting (17) into (15) and performing some obvious simplifications leads to (20). The reason why $b\left(s_{t}\right)$ has to satisfy the boundary conditions (21) and (22) follows from the next proposition (proposition 6). Since (17) satisfies (15) for $b\left(s_{t}\right)$ solving (20), a classical verification theorem can be invoked to show that it must be the value function as long as the "tail" condition (61) is satisfied (Fleming and Soner (1993) p.172).

The above proposition is a "place-holder" for all the properties that need to be verified in order to establish that (17) is the value function of the problem. We start by showing that the boundaries to $b$ are given by $(21)$ and $(22)$.

Proposition 6 The value function of the problem with sudden stops for $s>\underline{s}$ satisfies:

$$
\begin{aligned}
& \lim _{s \rightarrow \infty} V\left(X_{\tau}, s, y_{\tau}^{*}\right)=\left(\frac{1}{r}\right)^{\gamma} \frac{\left(X_{\tau}+\frac{y_{\tau}^{*}}{r-g}\right)^{1-\gamma}}{1-\gamma} \\
& \lim _{s \rightarrow-\infty} V\left(X_{\tau}, s, y_{\tau}^{*}\right)=K\left(\frac{1}{r}\right)^{\gamma} \frac{\left(X_{\tau}+\frac{y_{\tau}^{*}}{r-g}\right)^{1-\gamma}}{1-\gamma}
\end{aligned}
$$

Proof. We focus on the $\gamma>1$ case. The proof proceeds in two steps. First one obtains an upper bound on the value function of the problem with sudden stops, which in this case is naturally given by the value function of the problem in the absence of sudden stops:

$$
V^{N S S}=\left(\frac{1}{r}\right)^{\gamma} \frac{\left(X_{\tau}+\frac{y_{\tau}^{*}}{r-g}\right)^{1-\gamma}}{1-\gamma}
$$


It tuns out that this expression is also a lower bound to the value function when $s_{t}$ goes to infinity. The lower bound is established upon observing that the consumption policy in the absence of sudden stops $c_{t}^{* N S S}, 0<t<\tau$ is still a feasible consumption /portfolio plan in the presence of sudden stops (since it satisfies the intertemporal budget constraint and also $X_{t}>-\frac{y_{t}^{*}}{r-g}$ for all $t$ ). Accordingly we have the two inequalities:

$$
V\left(X_{t}, s_{t}, y_{t}^{*}\right) \geq E_{t}\left[\int_{t}^{\tau} e^{-r(u-t)} \frac{\left(c_{u}^{* N S S}\right)^{1-\gamma}}{1-\gamma} d u+e^{-r(\tau-t)} 1\{\tau<\infty\} K\left(\frac{1}{r}\right)^{\gamma} \frac{\left(X_{\tau}^{N S S}+\frac{y_{\tau}^{*}}{r-a}\right)^{1-\gamma}}{1-\gamma}\right]
$$

However by standard arguments it is easy to show that the right hand side of the above equation goes to $V^{N S S}$ as $s \rightarrow \infty$. To see that, note that $\operatorname{Pr}(\tau<Q) \rightarrow 0$ for all finite $Q$, and thus

$$
\begin{gathered}
\lim _{s \rightarrow \infty} E\left[\int_{t}^{\tau} e^{-r(u-t)} \frac{\left(c_{u}^{* N S S}\right)^{1-\gamma}}{1-\gamma} d u+e^{-r(\tau-t)} K 1\{\tau<\infty\}\left(\frac{1}{r}\right)^{\gamma} \frac{\left(X_{\tau}^{N S S}+\frac{y_{\tau}^{*}}{r-a}\right)^{1-\gamma}}{1-\gamma} \mid F_{t}\right]= \\
=E\left[\int_{t}^{\infty} e^{-r(u-t)} \frac{\left(c_{u}^{* N S S}\right)^{1-\gamma}}{1-\gamma} d u \mid F_{t}\right]=V^{N S S}\left(X_{t}, y_{t}^{*}\right)= \\
\left(\frac{1}{r}\right)^{\gamma} \frac{\left(X_{t}+\frac{y_{t}^{*}}{r-g}\right)^{1-\gamma}}{1-\gamma}
\end{gathered}
$$

Since the upper and the lower bound coincide to $V^{N S S}$ as $s \rightarrow \infty$, the claim is established. A similar claim can be used to show the second result in the proposition.

Showing formally that $b\left(s_{t}\right)$ possesses a unique solution subject to the two boundary conditions (21) and (22) is a fairly hard mathematical task that is well beyond the scope of the present paper. The next Proposition provides a sketch of how one would proceed to obtain a proof. For the substantive results of the paper, we were always able to obtain numerically a solution to this ODE at arbitrary precision levels.

Proposition 7 There exists a solution to the ODE (20) subject to (21) and (22).

Proof. (Sketch) First we transform the second order ODE into a system of ODE's. Then we compute the steady states at $s=+\infty$ and $s=-\infty$, linearize the system and compute its eigenvalues. Using the stable manifold theorem around $s=0$ twice (as $s \rightarrow+\infty$ and as $s \rightarrow-\infty$ ) establishes the result. For more details see Caballero and Panageas (2003) Proposition 3. 
The last step in order to verify that (17) is indeed the Value function is to verify (61). To show (61) we need two Lemmas:

Lemma 4 Consider a consumption policy that has the feedback form:

$$
c_{t}^{*}=A\left(s_{t}\right)\left(X_{t}+\frac{y_{t}^{*}}{r-g}\right), \quad K^{-1 / \gamma} r \leq A\left(s_{t}\right) \leq r \quad \forall s_{t} \in(-\infty,+\infty)
$$

Then $X_{t} \geq X_{t}^{N S S}$, where $X_{t}^{N S S}$ is the asset process that results when one uses the optimal consumption policy in the absence of sudden stops:

$$
c_{t}^{N S S}=r\left(X_{t}^{N S S}+\frac{y_{t}^{*}}{r-g}\right)
$$

Proof. This result is trivial. One solves for the asset process that results from the two policies to find that

$$
X_{t}^{N S S}=X_{0}-\frac{y_{0}^{*}}{r-g}\left(e^{g t}-1\right)
$$

The equivalent calculation for the optimal policy $c_{t}$ conditional on a path of $s_{t}$ gives:

$$
\begin{aligned}
d\left(e^{-\left(r t-\int_{0}^{t} A\left(s_{u}\right) d u\right)} X_{t}\right) & =-\left(r-A\left(s_{t}\right)\right) e^{-\left(r t-\int_{0}^{t} A\left(s_{u}\right) d u\right)} X_{t}+e^{-\left(r t-\int_{0}^{t} A\left(s_{u}\right) d u\right)} d X_{t}= \\
& =e^{-\left(r t-\int_{0}^{t} A\left(s_{u}\right) d u\right)}\left[y_{0}^{*} \frac{r-A\left(s_{t}\right)-g}{r-g} e^{g t}\right]
\end{aligned}
$$

Now integrating both sides and rearranging gives:

$$
\begin{aligned}
X_{t}= & X_{0}+\frac{y_{0}^{*}}{r-g} e^{\left(r t-\int_{0}^{t} A\left(s_{u}\right) d u\right)}\left(\int_{0}^{t} e^{-\left((r-g) i-\int_{0}^{i} A\left(s_{u}\right) d u\right)}\left(r-A\left(s_{i}\right)-g\right) d i\right)= \\
& X_{0}-\frac{y_{0}^{*}}{r-g}\left(e^{g t}-e^{\left(r t-\int_{0}^{t} A\left(s_{u}\right) d u\right)}\right)
\end{aligned}
$$

The result now follows since $A\left(s_{t}\right) \leq r \quad \forall s_{t} \in(-\infty,+\infty)$.

Lemma 5 The function $b(s)$ is bounded between $\frac{1}{r} K^{1 / \gamma}$ and $\frac{1}{r}$.

Proof. We will derive the result by means of a contradiction. Suppose $b(s)$ solves (20) subject to the boundary conditions $(21),(22)$. Since $b(s)$ asymptotes to $\frac{1}{r}$ as $s \rightarrow \infty$ and to $\underline{b}$ as $s \rightarrow-\infty$ this means that if there exists a $b>\underline{b}$ then there must be a $s^{*}$ such that $b\left(s^{*}\right)>\underline{b}$ and $b\left(s^{*}\right)$ is a local max. Similarly if there exists a $b<\frac{1}{r}$ then there must be a $s^{*}$ such that $b\left(s^{*}\right)<\frac{1}{r}$ is a local 
min. Both are impossible though. We establish the first contradiction. The second is obtained similarly. Indeed, suppose that there exists one point $s^{*}$ such that $b_{s}\left(s^{*}\right)=0$ and $b\left(s^{*}\right)>\underline{b}$. Then (20) becomes:

$$
1-r b\left(s^{*}\right)+\frac{b\left(s^{*}\right)}{\gamma} \lambda\left(s^{*}\right)\left[\left(\frac{\underline{b}}{b\left(s^{*}\right)}\right)^{\gamma}-1\right]=-b_{s s} \frac{1}{2} \sigma^{2}
$$

which in turn implies that $b_{s s}\left(s^{*}\right)>0$ since the left hand side of the above equation is negative. Hence every extremum in the area $b\left(s^{*}\right)>\underline{b}$ must be a local minimum, and cannot be a maximum. Similarly if $b\left(s^{*}\right)<\frac{1}{r}$, a symmetric argument can be used, to show that is impossible to have a local min in this region.

Now, we are in a position to establish the "tail condition" (61):

Lemma 6 Assume $\left(\frac{1}{r}\right)^{\gamma} \leq b\left(s_{t}\right)^{\gamma} \leq K\left(\frac{1}{r}\right)^{\gamma} \forall s_{t} \in(-\infty,+\infty)$. Then

$$
\lim _{t \rightarrow \infty} E\left[e^{-r t} 1\{\tau>t\} b\left(s_{t}\right)^{\gamma} \frac{\left(X_{t}^{o p t}+\frac{y_{t}^{*}}{r-g}\right)^{1-\gamma}}{1-\gamma}\right]=0
$$

Proof. For any $t$ it is the case that

$$
\begin{gathered}
E\left[e^{-r t} 1\{\tau>t\} b\left(s_{t}\right)^{\gamma} \frac{\left(X_{t}^{o p t}+\frac{y_{t}^{*}}{r-g}\right)^{1-\gamma}}{1-\gamma}\right] \geq \\
E\left[e^{-r t} 1\{\tau>t\} K\left(\frac{1}{r}\right)^{\gamma} \frac{\left(X_{t}^{o p t}+\frac{y_{t}^{*}}{r-g}\right)^{1-\gamma}}{1-\gamma}\right] \geq \\
K E\left[e^{-r t} 1\{\tau>t\}\left(\frac{1}{r}\right)^{\gamma} \frac{\left(X_{t}^{N S S}+\frac{y_{t}^{*}}{r-g}\right)^{1-\gamma}}{1-\gamma}\right] \rightarrow 0
\end{gathered}
$$

The first inequality follows from the assumption and the fact that $\frac{\left(X_{t}^{o p t}+\frac{y_{t}^{*}}{r-g}\right)^{1-\gamma}}{1-\gamma}$ is a negative number. The second from the previous lemma and the monotonicity of the value function and the last limit follows from the fact that one can trivially show that in the standard model without sudden stops $e^{-r t}\left(\frac{1}{r}\right)^{\gamma} \frac{\left(X_{t}^{N S S}+\frac{y_{t}^{*}}{r-g}\right)^{1-\gamma}}{1-\gamma} \rightarrow 0$. To establish the result observe also that

$$
E\left[e^{-r t} 1\{\tau>t\} b\left(s_{t}\right)^{\gamma} \frac{\left(X_{t}^{o p t}+\frac{y_{t}^{*}}{r-g}\right)^{1-\gamma}}{1-\gamma}\right] \leq 0
$$

irrespective of $t$, since $\gamma>1$. Hence, it must be the case that (63) holds. 


\section{A.4 Proofs for section 4}

For all the value functions in this section one can apply similar verification arguments to the ones given in the previous section. Hence, we just focus on the substantive proofs of the Lemmas and the Propositions in the text.

Proof. (Lemma 3) The proof is a straightforward application of Ito's Lemma in conjunction with (27)

$$
\begin{aligned}
\frac{d c^{*}}{c^{*}}= & \frac{c_{X}}{c} d X_{t}+\frac{c_{y^{*}}}{c} d y_{t}^{*}+\frac{p_{t}^{2} \sigma^{2}}{2} \frac{c_{X X}}{c} d t+\frac{c_{s}}{c} d s+\frac{\sigma^{2}}{2} \frac{c_{s s}}{c} d t+p_{t} \sigma^{2} \frac{c_{X s}}{c} d t= \\
= & {\left[\left(r-\frac{1}{d}\right)-\lambda\left(s_{t}\right)\left(1+\phi_{2}\right) i-\frac{d_{s}}{d} \mu+\frac{\sigma^{2}}{2}\left[2\left(\frac{d_{s}}{d}\right)^{2}-\frac{d_{s s}}{d}\right]-\sigma^{2}\left(\frac{1}{\gamma} \frac{\phi_{1}}{\sigma^{2}}+\frac{d_{s}}{d}\right) \frac{d_{s}}{d}\right] d t } \\
& +\left(\frac{1}{\gamma} \frac{\phi_{1}}{\sigma}\right) d B_{t}
\end{aligned}
$$

The claim now follows directly after some obvious cancellations

Proof. (Proposition 1). For $\phi_{1}=\phi_{2}=0$ equation (37) becomes:

$$
\frac{d c^{*}}{c^{*}}=-\frac{1}{d}\left[1-r d+\lambda\left(s_{t}\right) i d+\mu d_{s}+\frac{\sigma^{2}}{2} d_{s s}\right] d t
$$

Notice also that for $\phi_{1}=\phi_{2}=0$, equation (36) becomes:

$$
0=1-r d+\mu d_{s}+\frac{\sigma^{2}}{2} d_{s s}+\lambda\left(s_{t}\right)\left\{\left(\frac{K^{\frac{1}{\gamma}}}{r}\right)-d\right\}
$$

so that:

$$
\frac{d c^{*}}{c^{*}}=-\frac{\lambda\left(s_{t}\right)}{d}\left\{i d-\left[\left(\frac{K^{\frac{1}{\gamma}}}{r}\right)-d\right]\right\} d t
$$

Note finally that $i$ is given by (34) as

$$
(1+i)=\frac{K^{1 / \gamma}}{r d}
$$

so that

$$
i d=\frac{K^{1 / \gamma}}{r}-d
$$

and therefore

$$
\frac{d c^{*}}{c^{*}}=-\frac{\lambda\left(s_{t}\right)}{d}\{i d-i d\} d t=0
$$


Proof. (Proposition 2) Similar to Propositions 3 and 4 (see below)

Proof. (Proposition 3) Suppose $\phi_{1}=0$, and that

$$
\frac{r d}{K^{1 / \gamma}}=\left(1+\phi_{2}\right)^{-\frac{1}{\gamma}}
$$

Then the Bellman equation (36) becomes:

$$
\begin{aligned}
0= & 1-r d+\mu d_{s}+\frac{\sigma^{2}}{2} d_{s s}+ \\
& +d \frac{1}{\gamma} \lambda\left(s_{t}\right)\left\{\left(\frac{K^{1 / \gamma}}{r d}\right)^{\gamma}-1\right\}
\end{aligned}
$$

and the consumption evolution (37) is:

$$
\begin{aligned}
\frac{d c^{*}}{c^{*}} & =-\frac{1}{d}\left[1-r d+d_{s} \mu+\frac{\sigma^{2}}{2} d_{s s}\right] d t= \\
& =-\frac{1}{d}\left[-d \frac{1}{\gamma} \lambda\left(s_{t}\right)\left\{\left(\frac{K^{1 / \gamma}}{r d}\right)^{\gamma}-1\right\}\right] d t= \\
& =\frac{1}{\gamma} \lambda\left(s_{t}\right)\left\{\left(\frac{K^{1 / \gamma}}{r d}\right)^{\gamma}-1\right\} d t
\end{aligned}
$$

Proof. (Proposition 4) Using (44) and (45) the Bellman equation (36) becomes:

$$
\begin{aligned}
0= & 1-r d+\mu d_{s}+\frac{\sigma^{2}}{2} d_{s s}+ \\
& -(1-\gamma) \frac{\sigma^{2}}{2} \frac{\left(d_{s}\right)^{2}}{d} \\
& +\frac{1}{\gamma} \lambda\left(s_{t}\right) d\left\{\left(\frac{K^{1 / \gamma}}{r d}\right)^{\gamma}-1\right\}
\end{aligned}
$$

The result then follows upon using Lemma 3. 